\title{
Frequency analysis of a uniform ring perturbed by point masses and springs
}

\author{
Amir H. Behbahani, Robert M'Closkey* \\ Mechanical and Aerospace Engineering Department, Samueli School of Engineering and Applied Science, \\ 420 Westwood Plaza, University of California, Los Angeles, CA 90095
}

\begin{abstract}
Perturbation expansions of solutions for a uniform, thin, linear elastic ring perturbed by point masses and radial massless springs are developed. The perturbation locations divide the ring into uniform segments so a variational formulation is used to determine the boundary conditions that must be satisfied between adjoining segments. The motion of each segment can be represented as a weighted sum of the eigenfunctions for the uniform thin ring so when the boundary conditions are enforced, the resulting algebraic relations are expanded as a function of the perturbation parameter (the perturbation mass normalized by the ring mass). A series of algebraic problems are sequentially solved to yield perturbation expansions for the modal frequencies and eigenmodes. Single-mass, dual-mass, and mass-spring case studies are considered. The perturbation results show excellent agreement with finite element analysis of a thin ring for mass perturbations up to $15 \%$ of the nominal ring mass. The results are also compared to Rayleigh-Ritz analysis.

Keywords: ring dynamics, vibratory gyro, perturbation analysis
\end{abstract}

\section{Introduction}

This paper studies the in-plane dynamics of a perturbed linearly elastic thin ring in which nonuniformities are created by point masses and massless radial springs. A perturbation approach is

\footnotetext{
*Corresponding author, Tel.: 0013108252909

Email addresses: amirbeseas . ucla . edu (Amir H. Behbahani), rtmeseas . ucla . edu (Robert M'Closkey)
} 


\section{Nomenclature}

A cross sectional area

E modulus of elasticity

$I \quad$ cross section moment of inertia

$M \quad$ uniform ring mass, $M=2 \pi R A \rho$

$\mathcal{N}(\mathbf{P}) \quad$ null space of matrix $\mathbf{P}$

$\mathcal{R}(\mathbf{P}) \quad$ range space of matrix $\mathbf{P}$

$\mathcal{R}(\mathbf{P})^{\perp} \quad$ orthogonal complement of $\mathcal{R}(\mathbf{P})$

$\operatorname{dim} \mathcal{S} \quad$ dimension of subspace $\mathcal{S}$

$\operatorname{rank}(\mathbf{P}) \quad$ rank of matrix $\mathbf{P}$

$\mathbf{P}^{T} \quad$ transpose of $\mathbf{P}$

$\mathrm{R} \quad$ ring mean radius

$\mathbf{R}^{n} \quad$ vector with $n$ real elements

$\mathbf{R}^{n \times m} \quad n \times m$ matrix with real elements $U$ radial displacement eigenfunction $\quad \kappa$

$W$

$W^{(p)}$

$\mathcal{L}$

$\mathcal{T}$

$\mathcal{U}$ tangential displacement eigenfunction $\omega$ $g_{1}$

$g_{2}$

j

K

$t$

$u$

$\mathbf{v}$

$\mathbf{v}_{k} \quad k$ th term in expansion for $\mathbf{v}$

$\mathbf{v}_{k_{l}} \quad l$ th partition of $\mathbf{v}_{k}$

$w$ tangential displacement

$\delta \quad$ variational derivative

$\epsilon \quad$ perturbation parameter

$\gamma \quad$ weight vector scaling parameter

$\kappa \quad$ time scale, $\kappa=\sqrt{\rho A R^{4} /(E I)}$

$\omega$ frequency

$\omega_{k} \quad k^{\text {th }}$ term in expansion for $\omega$

$\omega_{k_{l}} \quad k^{\text {th }}$ term in expansion for mode $l \in\{1,2\}$

$\rho \quad$ material density

$\theta \quad$ angle coordinate

used to determine expansions for the natural frequencies and eigenfunctions of the exact solutions of the perturbed ring. The perturbation parameter is the point mass value normalized by the unperturbed ring mass. Massless radial springs are also included in the analysis and their contribution relative to the point mass is quantified with an additional parameter. The perturbation results presented herein must be developed for specific scenarios, however, since the modes appear as degenerate pairs in the uniform ring, the common features among the various case studies are noted. The boundary conditions that demarcate uniform ring segments separated by the perturbation locations are developed using Hamilton's principle and the resulting algebraic constraints on the eigenfunction weights are expanded in the perturbation parameter and sequentially solved. The case studies consider single- and two-mass perturbations and a mass-spring perturbation. Frequency expansions are developed through order $-\epsilon^{3}$ and are shown to accurately predict the perturbed frequencies for 
large perturbations. Finite element analysis (FEA) of a thin ring are taken as the benchmark, and the results are also compared with Rayleigh-Ritz analysis using up to twenty basis functions.

The in-plane equations of motion for a ring were developed more than one century ago $[1,2]$ but analysis since the 1980's has focused on the dynamics of nonuniform rings since they represent more realistic structures. The analysis tool for most of these studies is the Rayleigh-Ritz method (eg. $[3,4,5,6])$ in which a certain number of eigenfunctions of the uniform ring are used as a solution basis. Other researchers have proposed more complicated basis functions for capturing the motion of an imperfect ring and allow general, distributed perturbations [7, 8]. For sufficiently small imperfections, many of these references also address the frequency trimming problem in which it is desired to reduce the frequency difference between a detuned pair of modes. The frequency trimming problem has recently received renewed interest from the sensor community because fabrication techniques now permit the creation of precision planar micro-scale resonant structures. For example, axisymmetric resonators have been recently reported [9, 10, 11, 12, 13]. The residual frequency difference after fabrication is large enough to warrant trimming, however, for these resonators the fabrication processes are still not sufficiently developed to permit manipulation of the resonator mass distribution so no experimental trimming results have been reported. Examples in which the experimental reduction of the modal frequency differences have been achieved are given in $[14,15]$. In the latter reference, the Rayleigh-Ritz analysis from [5] was adapted to create an iterative frequency trimming procedure that employs mass deposition on a planar microscale resonator consisting of multiple nested rings. Using the same resonator design, a tailored etch technique has also produced trimmed devices [16]. This paper does not address the frequency trimming procedure, however, it is shown in the case studies where comparison with finite elements is possible that accurate prediction of the perturbed natural frequencies is achieved for large perturbations. Connections with the frequency trimming problem will be pursued in the future and will have relevance to resonators with large initial differences between the pairs of model frequencies, eg. [17]. Although only the effect of point masses and radial restoring force springs are analyzed, they are good approximations of the mass deposition, targeted etch techniques and electrostatic 
"springs" that have been employed to modify the dynamics of micro-scale resonators.

The paper is organized as follows. Sec. 2 discusses the derivation of the boundary conditions created between ring segments due to point mass or radial spring perturbations. Sec. 3 solves the sixth order differential equation for a uniform ring in order to determine the complete set of eigenfunctions. Sec. 4 discusses the series of algebraic problems that are derived by expanding the relations between the eigenfunction weights in the perturbation parameter and considers case studies involving single mass, dual mass and mass-spring perturbations. Sec. 5 considers a thin silicon ring for the case studies and compares the perturbation results to Rayleigh-Ritz analysis and to finite elements. The ring parameters approximate a single ring of the resonator studied in [15]. Sec. 6 concludes the paper.

\section{Ring equation and boundary conditions}

The equation of motion and boundary conditions for a uniform ring that is perturbed by a point mass and a co-located massless spring exerting a radial force are derived using Hamilton's principle. The uniform ring is defined by the parameters $\rho, A, I, R$, and $M$ which are the material density, cross sectional area, cross section moment of inertia, mean radius and total mass of the ring, respectively. The ring's radial and tangential displacements, denoted $u(\theta, t)$ and $w(\theta, t)$, respectively, are dependent on the angle variable $\theta$ and the time variable $t$. The mass of the pointmass perturbation is $\epsilon g_{1} M$ and for the boundary condition derivations its location is taken to be $\theta=0$ without loss of generality, which is equivalent to $\theta=2 \pi$ radians due to the periodic structure of the ring (see Fig. 1). The spring stiffness is given by $\epsilon g_{2} K$. The parameter $\epsilon$ is used to generate a perturbation expansion of the exact solution of the perturbed ring, and the parameters $g_{1}$ and $g_{2}$ are used to modify the relative contributions of the perturbing mass and spring. The cases when there are more than one mass or spring perturbation can be simply extended from the analysis presented herein. Furthermore, if the mass and spring are not co-located then $g_{1}$ or $g_{2}$ can be set to zero to obtain the desired case. The standard thin ring equation of motion governs the segments between the perturbation locations and the derived boundary conditions specify relations 


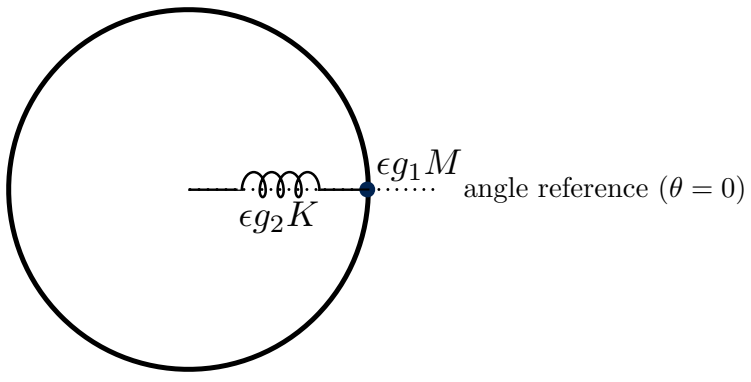

Figure 1: Point mass perturbation with co-located spring applied to a thin uniform ring.

between the solutions for each segment. In order to apply Hamilton's principle, the kinetic and strain energies for the Lagrangian functional must be determined.

The Lagrangian functional is $\mathcal{L}=\mathcal{T}-\mathcal{U}$, where $\mathcal{T}$ and $\mathcal{U}$ are the kinetic energy and strain energy, respectively. The kinetic energy $\mathcal{T}$ is composed of the kinetic energy of the ring and the kinetic energy of the mass perturbation located at $\theta=0$

$$
\mathcal{T}=\frac{1}{2} \int_{0}^{2 \pi} \rho A R\left(\dot{w}^{2}(\theta, t)+\dot{u}^{2}(\theta, t)\right) d \theta+\frac{1}{2} \epsilon g_{1} M\left((\dot{w}(0, t))^{2}+(\dot{u}(0, t))^{2}\right) .
$$

The strain energy of the ring is computed (see [18]) and summed with the strain energy of the spring,

$$
\mathcal{U}=\frac{1}{2} \int_{0}^{2 \pi} \frac{E I}{R^{3}}\left(\frac{\partial^{3} w}{\partial \theta^{3}}+\frac{\partial w}{\partial \theta}\right)^{2} d \theta+\frac{1}{2} \epsilon g_{2} K u^{2}(0, t) .
$$

The following small angle approximation is employed which provides a kinematic constraint between the radial and tangential velocities at a point: $u(\theta, t)=\frac{\partial w}{\partial \theta}(\theta, t)$.

Hamilton's principle is applied to the Lagrangian to determine the governing equation of motion and the boundary conditions. The Lagrangian functional variation is given in Appendix A where the equation of motion and boundary conditions are developed in terms of the tangential velocity $w$. The equation of motion is

$$
-\rho A R \ddot{w}(\theta, t)+\rho A R \frac{\partial^{2} \ddot{w}}{\partial \theta^{2}}(\theta, t)+\frac{E I}{R^{3}}\left(\frac{\partial^{6} w}{\partial \theta^{6}}+2 \frac{\partial^{4} w}{\partial \theta^{4}}+\frac{\partial^{2} w}{\partial \theta^{2}}\right)=0 .
$$

Harmonic ring motion is assumed so $w(\theta, t)=W(\theta) e^{\mathrm{j} \omega t}$, where $W$ denotes the mode shape associated with natural frequency $\omega$. The following notation is also used for the $p^{\text {th }}$ derivative of 
$W: W^{(p)}=\mathrm{d}^{p} W / \mathrm{d} \theta^{p}$. The essential boundary conditions given by Eqs. (A.2), (A.3) and (A.4) can be expressed in terms of $W$

$$
\begin{array}{r}
W(2 \pi)-W(0)=0, \\
W^{(1)}(2 \pi)-W^{(1)}(0)=0, \\
W^{(2)}(2 \pi)-W^{(2)}(0)=0 .
\end{array}
$$

The remaining natural boundary conditions given by Eqs. (A.6), (A.7) and (A.8) yield

$$
\begin{aligned}
W^{(3)}(2 \pi)-W^{(3)}(0) & =0 \\
\epsilon\left(\omega^{2} g_{1} M-g_{2} k\right) W^{(1)}(0)+\frac{E I}{R^{3}}\left(W^{(4)}(2 \pi)-W^{(4)}(0)\right) & =0 \\
\epsilon \omega^{2} g_{1} M W(0)-\frac{E I}{R^{3}}\left(W^{(5)}(2 \pi)-W^{(5)}(0)\right) & =0 .
\end{aligned}
$$

\section{Ring eigenfunctions}

The following differential equation is derived from Eq. (3) and may be solved for the ring eigenfunctions,

$$
W^{(6)}+2 W^{(4)}+W^{(2)}+\rho A \frac{R^{4}}{E I} \omega^{2}\left(W-W^{(2)}\right)=0 .
$$

The characteristic equation associated with Eq. (6) is

$$
\lambda^{6}+2 \lambda^{4}+\left(1-\rho A \frac{R^{4}}{E I} \omega^{2}\right) \lambda^{2}+\rho A \frac{R^{4}}{E I} \omega^{2}=0,
$$

in which only the even powers of $\lambda$ are present. A natural change of variable is $\alpha=\lambda^{2}$, which converts Eq. (7) into a third order equation for which closed-form solutions exist,

$$
\alpha^{3}+2 \alpha^{2}+\left(1-(\kappa \omega)^{2}\right) \alpha+(\kappa \omega)^{2}=0
$$

where $\kappa=\sqrt{\rho A \frac{R^{4}}{E I}}$. Note that $\kappa \omega$ represents a non-dimensional frequency. The discriminant of Eq. (8) is

$$
\Delta=36\left(1-(\kappa \omega)^{2}\right)(\kappa \omega)^{2}-32(\kappa \omega)^{2}+4\left(1-(\kappa \omega)^{2}\right)^{2}-4\left(1-(\kappa \omega)^{2}\right)^{3}-27(\kappa \omega)^{4},
$$


and has three real roots

$$
\begin{aligned}
& (\kappa \omega)^{2}=0, \\
& (\kappa \omega)^{2}=\frac{71 \pm 17 \sqrt{17}}{8} .
\end{aligned}
$$

The discriminant is positive $(\Delta>0)$ when $0<(\kappa \omega)^{2}<(71-17 \sqrt{17}) / 8$, or $(\kappa \omega)^{2}>(71+$ $17 \sqrt{17}) / 8$, and it is negative $(\Delta<0)$ when $(71-17 \sqrt{17}) / 8<(\kappa \omega)^{2}<(71+17 \sqrt{17}) / 8$.

\subsection{Eigenfunctions for $n=2$}

The sign of the discriminant determines different families of eigenfunctions, however, for the uniform thin ring, all eigenfunctions are of the form $\cos (n \theta+\psi)$, where $n=2,3,4, \ldots$, and $\psi$ is an arbitrary phase. The closed-form expression for the ring natural frequencies is $\omega^{2}=$ $\frac{n^{6}-2 n^{4}+n^{2}}{n^{2}+1} \frac{E I}{\rho A R^{4}}[19]$. Thus, for $n=2$, there exists a degenerate pair of modes with natural frequency $\omega_{0}^{2}=\frac{36 E I}{5 \rho A R^{4}}$. Subsituting $\left(\kappa \omega_{0}\right)^{2}=36 / 5$ into Eq. (9) shows that the discriminant is negative when $n=2$, which implies Eq. (8) has one real negative root and a complex conjugate pair of roots. Thus, in the perturbed ring, the six roots of Eq. (7) can be parameterized as $\{ \pm \mathrm{j} a, \pm b \pm \mathrm{j} c\}$, where $a, b$ and $c$ are real-valued parameters that are dependent on the perturbed natural frequency $\omega$. The corresponding eigenfunctions from Eq. (6) are given by $W(\theta)=e^{ \pm \mathrm{j} a \theta}$ and $W(\theta)=e^{( \pm b \pm \mathrm{j} c) \theta}$, which can be equivalently expressed as the following set

$$
\begin{array}{ll}
W_{1}(\theta)=\cos (a \theta), & W_{4}(\theta)=\cosh (b \theta) \sin (c \theta), \\
W_{2}(\theta)=\sin (a \theta), & W_{5}(\theta)=\sinh (b \theta) \cos (c \theta), \\
W_{3}(\theta)=\cosh (b \theta) \cos (c \theta), & W_{6}(\theta)=\sinh (b \theta) \sin (c \theta) .
\end{array}
$$

The perturbed natural frequencies are represented by regular perturbation expansions in the parameter $\epsilon$, in other words,

$$
\omega=\omega_{0}+\epsilon \omega_{1}+\epsilon^{2} \omega_{2}+\cdots
$$


Using this representation, the roots of Eq. (7) are also expressed as perturbation expansions. The series for the $a, b$ and $c$ parameters are determined to be

$$
\begin{array}{rl}
a=2+ & \epsilon\left(0.26001 \kappa \omega_{1}\right)+\epsilon^{2}\left(-0.019181 \kappa^{2} \omega_{1}^{2}+0.26001 \kappa \omega_{2}\right) \\
& +\epsilon^{3}\left(0.26001 \kappa \omega_{3}-0.038361 \kappa^{2} \omega_{1} \omega_{2}+0.0030343 \kappa^{3} \omega_{1}^{3}\right)+\cdots \\
b= & 1.0820+\epsilon\left(0.19537 \kappa \omega_{1}\right)+\epsilon^{2}\left(-0.025498 \kappa^{2} \omega_{1}^{2}+0.19537 \kappa \omega_{2}\right) \\
& +\epsilon^{3}\left(0.19537 \kappa \omega_{3}-0.050995 \kappa^{2} \omega_{1} \omega_{2}+0.0059897 \kappa^{3} \omega_{1}^{3}\right)+\cdots \\
c=0 & 01330+\epsilon\left(-0.11761 \kappa \omega_{1}\right)+\epsilon^{2}\left(-0.031796 \kappa^{2} \omega_{1}^{2}-0.11761 \kappa \omega_{2}\right) \\
& +\epsilon^{3}\left(-0.11761 \kappa \omega_{3}-0.063591 \kappa^{2} \omega_{1} \omega_{2}-0.0067277 \kappa^{3} \omega_{1}^{3}\right)+\cdots
\end{array}
$$

Symbolic computation software is used for these calculations [20].

The general solution for the perturbed ring is a weighted sum of the eigenfunctions in Eq. (10), in other words, $W(\theta)=\sum_{p=1}^{6} v_{p} W_{p}(\theta)$, where the set of weights $\left\{v_{p}\right\}$ will be determined by enforcing the constraints in Eq. (4) and Eq. (5). The process for finding these weights, and values for $\omega_{1}, \omega_{2}$ and $\omega_{3}$ are described in Sec. 4.

\subsection{Eigenfunctions for $n>2$}

When $n>2$, the discriminant is positive and Eq. (8) has three real roots, one of which is negative. Thus, the roots of Eq. (7) can be parameterized as $\{ \pm \mathrm{j} a, \pm b, \pm c\}$ which yields the following eigenfunctions,

$$
\begin{array}{ll}
W_{1}(\theta)=\cos (a \theta), & W_{4}(\theta)=\sinh (b \theta), \\
W_{2}(\theta)=\sin (a \theta), & W_{5}(\theta)=\cosh (c \theta), \\
W_{3}(\theta)=\cosh (b \theta), & W_{6}(\theta)=\sinh (c \theta) .
\end{array}
$$

Just as for the $n=2$ case, the parameters $a, b$ and $c$ can be written as a perturbation expansion in $\epsilon$. These expansions are given in Appendix B for $n=3,4,5$. 


\section{Perturbation expansions of solutions}

This section develops perturbation expansions of the solutions for number of perturbation scenarios. The first case, a single mass perturbation and no spring perturbation, is developed in Sec. 4.1. The details are given for $n=2$ modes in Sec. 4.1.1, and the $n=3$ modes are studied in Sec. 4.1.2. Dual-mass perturbation scenarios are studied in Sec. 4.2 and a non co-located spring-mass perturbation is discussed in Sec. 4.3.

When there are $p>0$ distinct perturbation locations, then there are $p$ ring segments governed by Eq. (3), so each segment can be expressed as a sum of the appropriate eigenfunctions (either Eq. (10) or Eq. (13)) with an associated weight vector. Thus, there are $6 p$ constraints generated by enforcing boundary conditions between each segment for a total of $6 p$ weights. Since the weights appear linearly in the constraints, the constraint equations can be generically written as $\mathbf{A v}=\mathbf{0}$ where $\mathbf{A} \in \mathbf{R}^{6 p \times 6 p}$ and the weights are collected into the vector $\mathbf{v} \in \mathbf{R}^{6 p}$. A perturbation expansion of the expressions for the modal frequencies and eigenfunctions is derived by expanding $\mathbf{A}$ and $\mathbf{v}$ with respect to $\epsilon: \mathbf{A}=\mathbf{A}_{0}+\epsilon \mathbf{A}_{1}+\epsilon^{2} \mathbf{A}_{2}+\cdots$, where

$$
\mathbf{A}_{0}=\left.\mathbf{A}\right|_{\epsilon=0}, \quad \mathbf{A}_{1}=\left.\frac{\partial \mathbf{A}}{\partial \epsilon}\right|_{\epsilon=0}, \quad \mathbf{A}_{2}=\left.\frac{1}{2} \frac{\partial^{2} \mathbf{A}}{\partial \epsilon^{2}}\right|_{\epsilon=0}, \ldots
$$

and $\mathbf{v}=\mathbf{v}_{0}+\epsilon \mathbf{v}_{1}+\epsilon^{2} \mathbf{v}_{2}+\cdots$, so

$$
\left(\mathbf{A}_{0}+\epsilon \mathbf{A}_{1}+\epsilon^{2} \mathbf{A}_{2}+\ldots\right)\left(\mathbf{v}_{0}+\epsilon \mathbf{v}_{1}+\epsilon^{2} \mathbf{v}_{2}+\ldots\right)=\mathbf{0} .
$$

$\mathbf{A}_{k}$ depends on frequency expansion terms $\omega_{p}, p=0,1, \ldots, k$. Thus, the following set of hierarchical algebraic problems is solved for every perturbation scenario

$$
\begin{array}{r}
\mathbf{A}_{0}\left(\omega_{0}\right) \mathbf{v}_{0}=\mathbf{0} \\
\mathbf{A}_{0}\left(\omega_{0}\right) \mathbf{v}_{1}+\mathbf{A}_{1}\left(\omega_{0}, \omega_{1}\right) \mathbf{v}_{0}=\mathbf{0} \\
\mathbf{A}_{0}\left(\omega_{0}\right) \mathbf{v}_{2}+\mathbf{A}_{1}\left(\omega_{0}, \omega_{1}\right) \mathbf{v}_{1}+\mathbf{A}_{2}\left(\omega_{0}, \omega_{1}, \omega_{2}\right) \mathbf{v}_{0}=\mathbf{0} \\
\mathbf{A}_{0}\left(\omega_{0}\right) \mathbf{v}_{3}+\mathbf{A}_{1}\left(\omega_{0}, \omega_{1}\right) \mathbf{v}_{2}+\mathbf{A}_{2}\left(\omega_{0}, \omega_{1}, \omega_{2}\right) \mathbf{v}_{1}+\mathbf{A}_{3}\left(\omega_{0}, \omega_{1}, \omega_{2}, \omega_{3}\right) \mathbf{v}_{0}=\mathbf{0}
\end{array}
$$


The solution details depend on the particular nature of the perturbations, however, there are some features common to all cases. It is generally possible to derive closed-form expressions for $\omega_{k}$ by projecting the columns of $\mathbf{A}_{k}$ onto the orthogonal complements, or the intersections thereof, of the subspaces $\left\{\mathcal{R}\left(\mathbf{A}_{0}\right), \mathcal{R}\left(\mathbf{A}_{1}\right), \ldots, \mathcal{R}\left(\mathbf{A}_{k-1}\right)\right\}$ and then selecting $\omega_{k}$ so that these projected components are zero. For example, the unperturbed ring possesses degenerate pairs of modes so $\operatorname{dim} \mathcal{R}\left(\mathbf{A}_{0}\right)^{\perp}=2$ and $\operatorname{dim} \mathcal{N}\left(\mathbf{A}_{0}\right)=2$, independent of the number of perturbations. In the case studies that follow, basis vectors for $\mathcal{R}\left(\mathbf{A}_{0}\right)^{\perp}$ are represented by the two columns of $\mathbf{P}_{0}$. Multiplying Eq. (16) on the left by $\mathbf{P}_{0}^{T}$ and restricting $\mathbf{v}_{0}$ to $\mathcal{N}\left(\mathbf{A}_{0}\right)$ yields a $2 \times 2$ matrix whose determinant must be zero. The entries of this matrix are affine in $\omega_{1}$ because the elements of $\mathbf{A}_{1}$ are affine functions of $\omega_{1}$. Thus, simple expressions can be derived for determining $\omega_{1}$. In fact, as $\mathbf{A}_{k}$ is an affine function of $\omega_{k}$, expressions for $\omega_{k}$ are at most quadratic. In the case studies that follow, the natural frequency expansions are computed through order $\epsilon^{3}$.

\subsection{Single mass perturbations}

4.1.1. $n=2$ case

Consider the case of a single point mass perturbation $\left(g_{1}=1, g_{2}=0\right)$ located at $\theta=0$. For the $n=2$ modes, the constraints in Eq. (4) and Eq. (5) yield six equations for the six to-be-determined weights $\left\{v_{1}, \ldots, v_{6}\right\}$ associated with the $n=2$ eigenfunctions as shown below

$$
\begin{array}{ll}
\sum_{p} v_{p}\left(W_{p}(2 \pi)-W_{p}(0)\right)=0, & \sum_{p} v_{k}\left(W_{p}^{(1)}(2 \pi)-W_{p}^{(1)}(0)\right)=0, \\
\sum_{p} v_{p}\left(W_{p}^{(2)}(2 \pi)-W_{p}^{(2)}(0)\right)=0, & \sum_{p} v_{k}\left(W_{p}^{(3)}(2 \pi)-W_{p}^{(3)}(0)\right)=0, \\
\sum_{p} v_{p}\left(\epsilon M \omega^{2} W_{p}^{(1)}(0)+\frac{E I}{R^{3}}\left(W_{p}^{(4)}(2 \pi)-W_{p}^{(4)}(0)\right)\right)=0, \\
\sum_{p} v_{p}\left(\epsilon M \omega^{2} W_{p}(0)-\frac{E I}{R^{3}}\left(W_{p}^{(5)}(2 \pi)-W_{p}^{(5)}(0)\right)\right)=0 .
\end{array}
$$

In this case, $\mathbf{A} \in \mathbf{R}^{6 \times 6}$ matrix derived from Eq. (19) and $\mathbf{v}=\left[v_{1}, \ldots, v_{6}\right]^{T} \in \mathbf{R}^{6}$ is the non-zero weight vector. For example, when the eigenfunctions are defined as shown in Eq. (10), the first row 
of $\mathbf{A}$ is

$$
\begin{array}{r}
{[1-\cos (2 \pi a),-\sin (2 \pi a), \quad 1-\cosh (2 \pi b) \cos (2 \pi c), \quad-\cosh (2 \pi b) \sin (2 \pi c),} \\
-\sinh (2 \pi b) \cos (2 \pi c), \quad-\sinh (2 \pi b) \sin (2 \pi c)] .
\end{array}
$$

As noted in Sec. 3, the parameters $a, b$ and $c$ are functions of $\epsilon$ because they are related to the roots of the perturbed characteristic polynomial (see Eq. (12)). Symbolic calculation yields the matrices $\mathbf{A}_{0}, \mathbf{A}_{1}, \mathbf{A}_{2}, \ldots$, but because of their structure, it is useful to partition these matrices as follows: $\mathbf{A}_{k}=\left[\begin{array}{ll}\mathbf{A}_{k_{1}} & \mathbf{A}_{k_{2}}\end{array}\right], k=0,1,2, \ldots$, where the $\mathbf{A}_{k_{1}} \in \mathbf{R}^{6 \times 2}$ and $\mathbf{A}_{k_{2}} \in \mathbf{R}^{6 \times 4}$. Similarly, $\mathbf{v}_{k}$ are partitioned into

$$
\mathbf{v}_{k}=\left[\begin{array}{l}
\mathbf{v}_{k_{1}} \\
\mathbf{v}_{k_{2}}
\end{array}\right], k=0,1,2, \ldots
$$

which are compatible with the partitions of $\mathbf{A}_{k}$. The analysis is general in the sense that the computed matrices $\mathbf{A}_{k}$ are common to all thin ring problems with a single mass perturbation in which the non-dimensional frequency parameters $\left\{\kappa \omega_{0}, \kappa \omega_{1}, \ldots\right\}$ are used. Thus, these computations need only be performed once to the desired accuracy.

The key steps are presented here, however, the numerical results for intermediate calculations can be found in Appendix C. The partitions of $\mathbf{A}_{0}$ (see Eq. (C.1)) are constant since they are functions of the known parameter $\kappa \omega_{0}=\sqrt{36 / 5}$. Since $\operatorname{rank}\left(\mathbf{A}_{0_{2}}\right)=4$, the first constraint, Eq. (15), yields $\mathbf{v}_{0_{2}}=\mathbf{0}$, however, $\mathbf{v}_{0_{1}}$ is undetermined. The next set of constraints, Eq. (16), yields

$$
\left[\begin{array}{ll}
\mathbf{0} & \mathbf{A}_{0_{2}}
\end{array}\right]\left[\begin{array}{l}
\mathbf{v}_{1_{1}} \\
\mathbf{v}_{1_{2}}
\end{array}\right]+\left[\begin{array}{ll}
\mathbf{A}_{1_{1}} & \mathbf{A}_{1_{2}}
\end{array}\right]\left[\begin{array}{c}
\mathbf{v}_{0_{1}} \\
\mathbf{0}
\end{array}\right]=\mathbf{0} \Longrightarrow\left[\begin{array}{ll}
\mathbf{A}_{1_{1}} & \mathbf{A}_{0_{2}}
\end{array}\right]\left[\begin{array}{l}
\mathbf{v}_{0_{1}} \\
\mathbf{v}_{1_{2}}
\end{array}\right]=\mathbf{0},
$$

where the known zero partitions have been inserted. Let the columns of $\mathbf{P}_{0} \in \mathbf{R}^{6 \times 2} \operatorname{span} \mathcal{R}\left(\mathbf{A}_{0_{2}}\right)^{\perp}$. Left-multiplication of Eq. (20) by $\mathbf{P}_{0}^{T}$ yields $\mathbf{P}_{0}^{T} \mathbf{A}_{1_{1}} \mathbf{v}_{0_{1}}=\mathbf{0}$, where

$$
\mathbf{P}_{0}^{T} \mathbf{A}_{1_{1}}=\left[\begin{array}{cc}
12.655+23.581 \kappa \omega_{1} & 18.786+8.7515 \kappa \omega_{1} \\
-9.3931-17.503 \kappa \omega_{1} & 25.309+11.790 \kappa \omega_{1}
\end{array}\right],
$$

and where $\mathbf{P}_{0}$ and $\mathbf{A}_{1_{1}}$ are given by Eq. (C.2) and Eq. (C.3), respectively. Non-trivial $\mathbf{v}_{0_{1}}$ will exist at those values of $\kappa \omega_{1}$ where $\operatorname{det}\left(\mathbf{P}_{0}^{T} \mathbf{A}_{1_{1}}\right)=\mathbf{0}$. These values, denoted $\kappa \omega_{1_{1}}$ and $\kappa \omega_{1_{2}}$, are

$$
\kappa \omega_{1_{1}}=-2.1466, \quad \kappa \omega_{1_{2}}=-0.53665 .
$$


At these two values of $\kappa \omega_{1}, \operatorname{rank}\left(\left[\begin{array}{ll}\mathbf{A}_{1_{1}} & \mathbf{A}_{0_{2}}\end{array}\right]\right)=5$, so solutions for $\mathbf{v}_{0_{1}}$ and $\mathbf{v}_{1_{2}}$, denoted $\tilde{\mathbf{v}}_{0_{1}}$ and $\tilde{\mathbf{v}}_{1_{2}}$, respectively, are unique up to a scaling,

$$
\begin{aligned}
& \kappa \omega_{1_{1}}=-2.1466 \Longrightarrow \tilde{\mathbf{v}}_{0_{1}}=\left[\begin{array}{l}
0 \\
1
\end{array}\right], \quad \tilde{\mathbf{v}}_{1_{2}}=\left[\begin{array}{llll}
-1.7534 & -9.7813 & 1.7614 & 9.8020
\end{array}\right]^{T}, \\
& \kappa \omega_{1_{2}}=-0.53665 \Longrightarrow \tilde{\mathbf{v}}_{0_{1}}=\left[\begin{array}{l}
1 \\
0
\end{array}\right], \quad \tilde{\mathbf{v}}_{1_{2}}=\left[\begin{array}{llll}
-2.2170 & -3.6827 & 2.2169 & 3.6731
\end{array}\right]^{T} .
\end{aligned}
$$

The $\tilde{\mathbf{v}}_{0_{1}}$ partitions are normalized to unit length and this sets the scaling for all subsequent elements of the series for the weight vector. Note that the leading order terms imply that the tangential motion for a perturbation at $\theta=0$ are close to $\sin (2 \theta)$ and $\cos (2 \theta)$. Thus, one radial antinode is essentially aligned with the perturbation location at $\theta=0^{\circ}$ and the other antinode is located near $45^{\circ}$.

The perturbed natural frequencies up to order $\epsilon^{2}$ can be computed from Eq. (17), rewritten below with the partitions,

$$
\left[\begin{array}{ll}
\mathbf{0} & \mathbf{A}_{0_{2}}
\end{array}\right]\left[\begin{array}{c}
\mathbf{v}_{2_{1}} \\
\mathbf{v}_{2_{2}}
\end{array}\right]+\left[\begin{array}{ll}
\mathbf{A}_{1_{1}} & \mathbf{A}_{1_{2}}
\end{array}\right]\left[\begin{array}{c}
\mathbf{v}_{1_{1}} \\
\tilde{\mathbf{v}}_{1_{2}}
\end{array}\right]+\left[\begin{array}{ll}
\mathbf{A}_{2_{1}} & \mathbf{A}_{2_{2}}
\end{array}\right]\left[\begin{array}{c}
\tilde{\mathbf{v}}_{0_{1}} \\
\mathbf{0}
\end{array}\right]=\mathbf{0}
$$

The unknown weights $\mathbf{v}_{2_{2}}$ and $\mathbf{v}_{1_{1}}$ are gathered into a single vector as follows,

$$
\left[\begin{array}{ll}
\mathbf{A}_{1_{1}} & \mathbf{A}_{0_{2}}
\end{array}\right]\left[\begin{array}{c}
\mathbf{v}_{1_{1}} \\
\mathbf{v}_{2_{2}}
\end{array}\right]+\left[\begin{array}{ll}
\mathbf{A}_{2_{1}} & \mathbf{A}_{1_{2}}
\end{array}\right]\left[\begin{array}{c}
\tilde{\mathbf{v}}_{0_{1}} \\
\tilde{\mathbf{v}}_{1_{2}}
\end{array}\right]=\mathbf{0}
$$

Since $\operatorname{rank}\left(\left[\begin{array}{ll}\mathbf{A}_{1_{1}} & \mathbf{A}_{0_{2}}\end{array}\right]\right)=5$ when $\omega_{1}=\left\{\omega_{1_{1}}, \omega_{1_{2}}\right\}$, let $\mathbf{P}_{1} \in \mathbf{R}^{6} \operatorname{span} \mathcal{R}\left(\left[\begin{array}{ll}\mathbf{A}_{1_{1}} & \mathbf{A}_{0_{2}}\end{array}\right]\right)^{\perp}$ when $\kappa \omega_{1}$ is selected as either value in Eq. (21). Left-multiplying Eq. (23) by $\mathbf{P}_{1}^{T}$ yields

$$
\mathbf{P}_{1}^{T}\left[\begin{array}{ll}
\mathbf{A}_{2_{1}} & \mathbf{A}_{1_{2}}
\end{array}\right]\left[\begin{array}{c}
\tilde{\mathbf{v}}_{0_{1}} \\
\tilde{\mathbf{v}}_{1_{2}}
\end{array}\right]=0
$$

When $\kappa \omega_{1_{1}}=-2.1466$, Eq. (24) yields $\kappa \omega_{2_{1}}=3.9104$, and when $\kappa \omega_{1_{2}}=-0.53666$ it yields $\kappa \omega_{2_{2}}=1.2159$. Expressions for both values of frequency deviations for $\mathbf{P}_{1}$ and $\mathbf{A}_{2_{1}}$ are given in Eqs. (C.5), (C.6), and Eq. (C.7), respectively. 
Solutions for $\left[\begin{array}{ll}\mathbf{v}_{1_{1}} & \mathbf{v}_{2_{2}}\end{array}\right]^{T}$ can now be determined from Eq. (23). Although, they are not unique they can be expressed as a unique least norm solution, denoted $\left[\begin{array}{ll}\tilde{\mathbf{v}}_{1_{1}} & \tilde{\mathbf{v}}_{2_{2}}\end{array}\right]^{T}$, plus an arbitrary vector in $\mathcal{N}\left(\left[\begin{array}{ll}\mathbf{A}_{1_{1}} & \mathbf{A}_{0_{2}}\end{array}\right]\right)$. From Eq. (20), a vector that spans the null space of this matrix is $\left[\begin{array}{ll}\tilde{\mathbf{v}}_{0_{1}} & \tilde{\mathbf{v}}_{1_{2}}\end{array}\right]^{T}$. Thus, the eigenfunction weights satisfying Eq. (23) are

$$
\left[\begin{array}{c}
\mathbf{v}_{1_{1}} \\
\mathbf{v}_{2_{2}}
\end{array}\right]=\left[\begin{array}{c}
\tilde{\mathbf{v}}_{1_{1}} \\
\tilde{\mathbf{v}}_{2_{2}}
\end{array}\right]+\gamma\left[\begin{array}{c}
\tilde{\mathbf{v}}_{0_{1}} \\
\tilde{\mathbf{v}}_{1_{2}}
\end{array}\right]
$$

where $\gamma$ is a real parameter. The least norm solution associated with $\kappa \omega_{1_{1}}$ is

$$
\left[\begin{array}{c}
\tilde{\mathbf{v}}_{1_{1}} \\
\tilde{\mathbf{v}}_{2_{2}}
\end{array}\right]=\left[\begin{array}{llllll}
1.7535 & 2.5193 & -1.5010 & 0.43081 & 1.4858 & -0.36263
\end{array}\right]^{T},
$$

and the least norm solution associated with $\kappa \omega_{1_{2}}$ is

$$
\left[\begin{array}{c}
\tilde{\mathbf{v}}_{1_{1}} \\
\tilde{\mathbf{v}}_{2_{2}}
\end{array}\right]=\left[\begin{array}{llllll}
2.3833 & -0.43836 & -0.30261 & 0.50506 & 0.30756 & -0.51076
\end{array}\right]^{T} .
$$

The frequency expansion is computed up to $\epsilon^{3}$ since this will yield accurate estimates of the perturbed modal frequencies even for relatively large perturbations. Expanding Eq. (18) into its partitions yields,

$$
\begin{aligned}
{\left[\begin{array}{ll}
\mathbf{0} & \mathbf{A}_{0_{2}}
\end{array}\right]\left[\begin{array}{c}
\mathbf{v}_{3_{1}} \\
\mathbf{v}_{3_{2}}
\end{array}\right]+\left[\begin{array}{ll}
\mathbf{A}_{1_{1}} & \mathbf{A}_{1_{2}}
\end{array}\right] } & {\left[\begin{array}{c}
\mathbf{v}_{2_{1}} \\
\tilde{\mathbf{v}}_{2_{2}}+\gamma \tilde{\mathbf{v}}_{1_{2}}
\end{array}\right] } \\
& +\left[\begin{array}{ll}
\mathbf{A}_{2_{1}} & \mathbf{A}_{2_{2}}
\end{array}\right]\left[\begin{array}{c}
\tilde{\mathbf{v}}_{1_{1}}+\gamma \tilde{\mathbf{v}}_{0_{1}} \\
\tilde{\mathbf{v}}_{12}
\end{array}\right]+\left[\begin{array}{ll}
\mathbf{A}_{3_{1}} & \mathbf{A}_{3_{2}}
\end{array}\right]\left[\begin{array}{c}
\tilde{\mathbf{v}}_{0_{1}} \\
\mathbf{0}
\end{array}\right]=\mathbf{0} .
\end{aligned}
$$

The unknown weights are $\mathbf{v}_{3_{1}}, \mathbf{v}_{3_{2}}$, and $\mathbf{v}_{2_{1}}$. Furthermore, $\mathbf{A}_{3_{1}}$ and $\mathbf{A}_{3_{2}}$ are functions of $\kappa \omega_{3}$. Leftmultiplying Eq. (26) by $\mathbf{P}_{1}^{T}$ (for a particular value of $\kappa \omega_{1}$ ), eliminates $\mathbf{v}_{3_{1}}, \mathbf{v}_{3_{2}}$ and $\mathbf{v}_{2_{1}}$ and produces an expression that is only a function of $\kappa \omega_{3}$,

$$
\mathbf{P}_{1}^{T} \mathbf{A}_{1_{2}} \tilde{\mathbf{v}}_{2_{2}}+\mathbf{P}_{1}^{T} \mathbf{A}_{2_{1}} \tilde{\mathbf{v}}_{1_{1}}+\gamma \underbrace{\mathbf{P}_{1}^{T}\left(\mathbf{A}_{1_{2}} \tilde{\mathbf{v}}_{1_{2}}+\mathbf{A}_{2_{1}} \tilde{\mathbf{v}}_{0_{1}}\right)}_{=0 \text {, per Eq. (24) }}+\mathbf{P}_{1}^{T} \mathbf{A}_{2_{2}} \mathbf{v}_{12}+\mathbf{P}_{1}^{T} \mathbf{A}_{3_{1}} \mathbf{v}_{0_{1}}=0 .
$$


Although $\mathbf{A}_{2_{2}}$ and $\mathbf{A}_{3_{1}}$ required to compute this expression, they are not given in Appendix $\mathrm{C}$ due to their length. Nevertheless, Eq. (27) is affine in $\kappa \omega_{3}$, and when $\kappa \omega_{1_{1}}=-2.1466$ and $\kappa \omega_{2_{1}}=$ 3.9104, Eq. (27) yields $\kappa \omega_{3_{1}}=-6.0317$. Similarly, when $\kappa \omega_{1_{2}}=-0.53665$ and $\kappa \omega_{2_{2}}=1.2159$, Eq. (27) yields $\kappa \omega_{3_{2}}=-2.7520$. Gathering the frequency terms yields the following expansions

$$
\begin{aligned}
& \kappa \omega=\sqrt{\frac{36}{5}}+\epsilon(-2.1466)+\epsilon^{2}(3.9104)+\epsilon^{3}(-6.0317)+\cdots, \\
& \kappa \omega=\sqrt{\frac{36}{5}}+\epsilon(-0.53665)+\epsilon^{2}(1.2159)+\epsilon^{3}(-2.7520)+\cdots .
\end{aligned}
$$

The role of $\gamma$ will now be clarified in Eq. (25). Note that left-multiplication of Eq. (26) by $\mathbf{P}_{1}^{T}$ eliminated $\mathbf{v}_{3_{1}}, \mathbf{v}_{3_{2}}, \gamma$, and $\mathbf{v}_{2_{1}}$. Define $\mathbf{P}_{2} \in \mathbf{R}^{6}$ such that $\mathbf{P}_{1}$ and $\mathbf{P}_{2}$ form a basis for $\mathcal{R}\left(\mathbf{A}_{0_{2}}\right)^{\perp}$ (this subspace is also spanned by the columns of $\mathbf{P}_{0}$ ). Then, left-multiplication of Eq. (26) by $\mathbf{P}_{2}^{T}$ also eliminates $\mathbf{v}_{3_{1}}$ and $\mathbf{v}_{3_{2}}$ but yields a relation between $\gamma$ and unknown vector $\mathbf{v}_{2_{1}}$,

$$
\mathbf{P}_{2}^{T} \mathbf{A}_{1_{1}} \mathbf{v}_{2_{1}}+\mathbf{P}_{2}^{T}\left(\mathbf{A}_{1_{2}} \tilde{\mathbf{v}}_{2_{2}}+\mathbf{A}_{2_{1}} \tilde{\mathbf{v}}_{1_{1}}+\mathbf{A}_{2_{2}} \tilde{\mathbf{v}}_{1_{2}}+\mathbf{A}_{3_{1}} \tilde{\mathbf{v}}_{1_{1}}\right)+\gamma \mathbf{P}_{2}^{T}\left(\mathbf{A}_{1_{2}} \tilde{\mathbf{v}}_{1_{2}}+\mathbf{A}_{2_{1}} \tilde{\mathbf{v}}_{0_{1}}\right)=0
$$

The solution for $\mathbf{v}_{2_{1}}$ can be written as a function of two parameters. First, let $\tilde{\mathbf{v}}_{2_{1}}$ represent the least-norm solution to Eq. (29) when $\gamma=0$, that is, $\tilde{\mathbf{v}}_{2_{1}}$ satisfies

$$
\mathbf{P}_{2}^{T} \mathbf{A}_{1_{1}} \tilde{\mathbf{v}}_{2_{1}}+\mathbf{P}_{2}^{T}\left(\mathbf{A}_{1_{2}} \tilde{\mathbf{v}}_{2_{2}}+\mathbf{A}_{2_{1}} \tilde{\mathbf{v}}_{1_{1}}+\mathbf{A}_{2_{2}} \tilde{\mathbf{v}}_{1_{2}}+\mathbf{A}_{3_{1}} \tilde{\mathbf{v}}_{0_{1}}\right)=0
$$

It can be shown that the dimension of $\mathcal{N}\left(\mathbf{P}_{2}^{T} \mathbf{A}_{1_{1}}\right)$ is one, so let $\mathbf{u}$ be its basis vector. All $\mathbf{v}_{2_{1}}$ solutions of Eq. (30) can be expressed as $\tilde{\mathbf{v}}_{2_{1}}+\alpha \mathbf{u}$, where $\alpha$ is a free parameter. If $\gamma \neq 0$, then $\tilde{\mathbf{v}}_{2_{1}}+\alpha \mathbf{u}+\gamma \tilde{\mathbf{v}}_{1_{1}}$ satisfies Eq. (29) because

$$
\mathbf{P}_{2}^{T}\left(\mathbf{A}_{1_{1}} \tilde{\mathbf{v}}_{1_{1}}+\mathbf{A}_{1_{2}} \tilde{\mathbf{v}}_{1_{2}}+\mathbf{A}_{2_{1}} \tilde{\mathbf{v}}_{0_{1}}\right)=0
$$

This is shown by first noting $\mathbf{P}_{2}^{T} \mathbf{A}_{1_{1}} \tilde{\mathbf{v}}_{0_{1}}=\mathbf{0}$. Then, left-multiplying Eq. (23) by $\mathbf{P}_{2}^{T}$,

$$
\begin{aligned}
& \mathbf{P}_{2}^{T}\left[\begin{array}{ll}
\mathbf{A}_{1_{1}} & \mathbf{A}_{0_{2}}
\end{array}\right]\left[\begin{array}{c}
\tilde{\mathbf{v}}_{1_{1}}+\gamma \tilde{\mathbf{v}}_{0_{1}} \\
\tilde{\mathbf{v}}_{2_{2}}+\gamma \tilde{\mathbf{v}}_{1_{2}}
\end{array}\right]+\mathbf{P}_{2}^{T}\left[\begin{array}{ll}
\mathbf{A}_{2_{1}} & \mathbf{A}_{1_{2}}
\end{array}\right]\left[\begin{array}{c}
\tilde{\mathbf{v}}_{0_{1}} \\
\tilde{\mathbf{v}}_{1_{2}}
\end{array}\right]=0 \\
\Longrightarrow & \mathbf{P}_{2}^{T} \mathbf{A}_{1_{1}} \tilde{\mathbf{v}}_{1_{1}}+\gamma \underbrace{\mathbf{P}_{2}^{T} \mathbf{A}_{1_{1}} \tilde{\mathbf{v}}_{0_{1}}}_{0}+\underbrace{\mathbf{P}_{2}^{T} \mathbf{A}_{0_{2}}}_{0}\left(\tilde{\mathbf{v}}_{2_{2}}+\gamma \tilde{\mathbf{v}}_{1_{2}}\right)+\mathbf{P}_{2}^{T}\left(\mathbf{A}_{2_{1}} \tilde{\mathbf{v}}_{0_{1}}+\mathbf{A}_{1_{2}} \tilde{\mathbf{v}}_{1_{2}}\right)=0,
\end{aligned}
$$


from which Eq. (31) follows. Gathering these results, the coefficient vector associated with a given frequency expansion is

$$
\mathbf{v}=\left[\begin{array}{c}
\tilde{\mathbf{v}}_{0_{1}} \\
\mathbf{0}
\end{array}\right]+\epsilon\left[\begin{array}{c}
\tilde{\mathbf{v}}_{1_{1}}+\gamma \tilde{\mathbf{v}}_{0_{1}} \\
\tilde{\mathbf{v}}_{1_{2}}
\end{array}\right]+\epsilon^{2}\left[\begin{array}{c}
\tilde{\mathbf{v}}_{2_{1}}+\alpha \mathbf{u}+\gamma \tilde{\mathbf{v}}_{1_{1}} \\
\tilde{\mathbf{v}}_{2_{2}}+\gamma \tilde{\mathbf{v}}_{1_{2}}
\end{array}\right]+\cdots
$$

which is rearranged to

$$
\mathbf{v}=(1+\epsilon \gamma)\left[\begin{array}{c}
\tilde{\mathbf{v}}_{0_{1}} \\
\mathbf{0}
\end{array}\right]+\epsilon(1+\epsilon \gamma)\left[\begin{array}{c}
\tilde{\mathbf{v}}_{1_{1}} \\
\tilde{\mathbf{v}}_{1_{2}}
\end{array}\right]+\cdots
$$

Although only the first few terms in the eigenfunction weights have been calculated, it appears that $\gamma$ merely scales the entire weight vector. So despite the fact that the leading order term $\left[\begin{array}{ll}\tilde{\mathbf{v}}_{0_{1}} & 0\end{array}\right]^{T}$ was normalized to have unit length, if $\gamma \neq 0$ the norm of this term is actually $1+\epsilon \gamma$, and the other terms are also scaled by this factor as well. Thus, $\gamma$ can be chosen to be zero without loss of generality.

\subsection{2. $n=3$ case}

The analysis for the $n=3$ pair of modes follows the same sequence of steps as the $n=2$ analysis with the exception that the eigenfunction basis is now given by Eq. (13) with the $a, b$ and $c$ parameters defined in Appendix B. Only the final results for the perturbed natural frequencies are given,

$$
\begin{aligned}
& \kappa \omega=\sqrt{\frac{576}{10}}+\epsilon(-6.8305)+\epsilon^{2}(20.553)+\epsilon^{3}(-42.001)+\cdots, \\
& \kappa \omega=\sqrt{\frac{576}{10}}+\epsilon(-0.75894)+\epsilon^{2}(1.9349)+\epsilon^{3}(-4.9136)+\cdots .
\end{aligned}
$$

Comparisons of the perturbed frequencies are made with FE and approximate methods in Sec. 5.

\subsection{Dual-mass perturbations}

The case in which two identical masses are placed approximately $45^{\circ}$ apart is addressed in this section. The analysis is performed for the $n=2$ modes. The modal frequencies detune for finite perturbations when the masses are exactly $45^{\circ}$ apart. Furthermore, when the masses are close to, but not exactly, $45^{\circ}$ apart, the modal frequencies initially detune as $\epsilon$ is increased, become degenerate, and then detune again. 


\subsubsection{Identical masses at $\theta=0$ and $\theta_{0}=44^{\circ}$}

The ring is divided into two segments when two mass perturbations are present and as remarked in the introduction to Sec. $4, \mathbf{A} \in \mathbf{R}^{12 \times 12}$ and $\mathbf{v} \in \mathbf{R}^{12}$. The locations of the perturbations are $\theta=0$ and $\theta_{0}=44^{\circ}$ (the angle origin coincides with one of the perturbations). The analysis that follows is also applicable to any two-mass perturbation when $\theta_{0} \neq 45^{\circ}$. The $\theta_{0}=45^{\circ}$ case is separately addressed in Sec. 4.2.2. The first 6 elements of $\mathbf{v}$ correspond to ring segment $\theta \in\left[0, \theta_{0}\right]$ and the last 6 elements of $\mathbf{v}$ correspond to the ring segment $\theta \in\left[\theta_{0}, 2 \pi\right]$ radians. With this parameterization, $\mathbf{A}_{0}$ is partitioned into four submatrices $\mathbf{A}_{0}=\left[\begin{array}{llll}\mathbf{A}_{0_{1}} & \mathbf{A}_{0_{2}} & \mathbf{A}_{0_{3}} & \mathbf{A}_{0_{4}}\end{array}\right]$, where $\mathbf{A}_{0_{1}}, \mathbf{A}_{0_{3}} \in \mathbf{R}^{12 \times 2}$, and $\mathbf{A}_{0_{2}}, \mathbf{A}_{0_{4}} \in \mathbf{R}^{12 \times 4}$. The partitions of $\mathbf{v}_{0}$ are similarly defined and compatible with the partitions of $\mathbf{A}_{0}: \mathbf{v}_{0}=\left[\begin{array}{llll}\mathbf{v}_{0_{1}} & \mathbf{v}_{0_{2}} & \mathbf{v}_{0_{3}} & \mathbf{v}_{0_{4}}\end{array}\right]^{T}$. The following can be shown

$$
\begin{aligned}
& \mathbf{A}_{0_{3}}=-\mathbf{A}_{0_{1}} \\
& \operatorname{rank}\left(\mathbf{A}_{0_{1}}\right)=2 \\
& \operatorname{rank}\left(\left[\begin{array}{lll}
\mathbf{A}_{0_{1}} & \mathbf{A}_{0_{2}} & \mathbf{A}_{0_{4}}
\end{array}\right]\right)=10
\end{aligned}
$$

Thus, $\operatorname{rank}\left(\mathbf{A}_{0}\right)=10$, and since the first constraint is $\mathbf{A}_{0} \mathbf{v}_{0}=\mathbf{0}$, this implies $\mathbf{v}_{0_{2}}=\mathbf{0}, \mathbf{v}_{0_{3}}=\mathbf{v}_{0_{1}}$, $\mathbf{v}_{0_{4}}=\mathbf{0}$, however, $\mathbf{v}_{0_{1}} \neq \mathbf{0}$ and is determined after further analysis. Eq. (16) is

$$
\left[\begin{array}{llll}
\mathbf{A}_{0_{1}} & \mathbf{A}_{0_{2}} & -\mathbf{A}_{0_{1}} & \mathbf{A}_{0_{4}}
\end{array}\right]\left[\begin{array}{c}
\mathbf{v}_{1_{1}} \\
\mathbf{v}_{1_{2}} \\
\mathbf{v}_{1_{3}} \\
\mathbf{v}_{1_{4}}
\end{array}\right]+\left[\begin{array}{llll}
\mathbf{A}_{1_{1}} & \mathbf{A}_{1_{2}} & \mathbf{A}_{1_{3}} & \mathbf{A}_{1_{4}}
\end{array}\right]\left[\begin{array}{c}
\mathbf{v}_{0_{1}} \\
\mathbf{0} \\
\mathbf{v}_{0_{1}} \\
\mathbf{0}
\end{array}\right]=\mathbf{0}
$$

where $\mathbf{v}_{1}$ is also partitioned as shown. Reusing notation from Sec. 4.1.1, let $\mathbf{P}_{0} \in \mathbf{R}^{12 \times 2}$ be defined such that its columns span $\mathcal{R}\left(\mathbf{A}_{0}\right)^{\perp}$. Left-multiplying Eq. (33) by $\mathbf{P}_{0}^{T}$ yields the following expression involving $\kappa \omega_{1}$,

$$
\mathbf{P}_{0}^{T}\left(\mathbf{A}_{1_{1}}+\mathbf{A}_{1_{3}}\right) \mathbf{v}_{0_{1}}=\left[\begin{array}{cc}
-33.888-12.714 \kappa \omega_{1} & -8.9157-3.5861 \kappa \omega_{1} \\
-9.6204-3.3234 \kappa \omega_{1} & 34.107+12.632 \kappa \omega_{1}
\end{array}\right] \mathbf{v}_{0_{1}}=\mathbf{0}
$$


Solutions for $\mathbf{v}_{0_{1}}$ will exist when the matrix multiplying it is singular. This yields the following values for $\kappa \omega_{1}$,

$$
\kappa \omega_{1_{1}}=-2.7395, \quad \kappa \omega_{1_{2}}=-2.6271
$$

at which the matrix is rank 1 . The values for $\kappa \omega_{1}$ are substituted into Eq. (33), which, after some rearrangement yields,

$$
\left[\begin{array}{llll}
\left(\mathbf{A}_{11}+\mathbf{A}_{1_{3}}\right) & \mathbf{A}_{0_{2}} & \mathbf{A}_{0_{1}} & \mathbf{A}_{0_{4}}
\end{array}\right]\left[\begin{array}{c}
\mathbf{v}_{0_{1}} \\
\mathbf{v}_{1_{2}} \\
\mathbf{v}_{1_{1}}-\mathbf{v}_{1_{3}} \\
\mathbf{v}_{1_{4}}
\end{array}\right]=\mathbf{0}
$$

and because the matrix multiplying the coefficient vector is rank 11, the solution vector is unique up to a scaling. The weight vectors associated with $\kappa \omega_{1_{1}}$ are

$$
\begin{aligned}
\tilde{\mathbf{v}}_{0_{1}} & =\left[\begin{array}{c}
-0.69466 \\
0.71934
\end{array}\right], \quad \tilde{\mathbf{v}}_{1_{2}}=\left[\begin{array}{llll}
0.76082 & -6.3518 & 0.23719 & 2.5828
\end{array}\right]^{T} \\
\tilde{\mathbf{v}}_{1_{1}}-\tilde{\mathbf{v}}_{1_{3}} & =\left[\begin{array}{c}
-0.69669 \\
-2.4999
\end{array}\right], \quad \tilde{\mathbf{v}}_{1_{4}}=\left[\begin{array}{llll}
1.0402 & -27.417 & -1.0332 & 27.443
\end{array}\right]^{T} .
\end{aligned}
$$

Similarly, for $\kappa \omega_{1_{2}}$ the solution vectors are

$$
\begin{aligned}
\tilde{\mathbf{v}}_{0_{1}} & =\left[\begin{array}{c}
-0.71934 \\
-0.69466
\end{array}\right], \quad \tilde{\mathbf{v}}_{1_{2}}=\left[\begin{array}{llll}
5.2538 & 5.9227 & -0.35983 & -12.985
\end{array}\right]^{T} \\
\tilde{\mathbf{v}}_{1_{1}}-\tilde{\mathbf{v}}_{1_{3}} & =\left[\begin{array}{c}
0.54526 \\
-2.4566
\end{array}\right], \quad \tilde{\mathbf{v}}_{1_{4}}=\left[\begin{array}{llll}
6.6055 & 0.65224 & -6.6116 & -0.65018
\end{array}\right]^{T} .
\end{aligned}
$$

The weight vectors are scaled so that the $\tilde{\mathbf{v}}_{0_{1}}$ partition has unit norm. The tangential displacement will be dominated by $W(\theta)=-0.69466 \cos (2 \theta)+0.71934 \sin (2 \theta)$ and $W(\theta)=0.71934 \cos (2 \theta)+$ $0.69466 \sin (2 \theta)$ which implies the radial displacement $U=\mathrm{d} W / \mathrm{d} \theta$ will be proportional to

$$
\begin{aligned}
& U(\theta) \propto 0.69466 \sin (2 \theta)+0.71934 \cos (2 \theta)=\cos (2(\theta-22 \pi / 180)) \\
& U(\theta) \propto-0.71934 \sin (2 \theta)+0.69466 \cos (2 \theta)=\cos (2(\theta+23 \pi / 180)) .
\end{aligned}
$$


Thus, one of the antinodes associated with the radial displacement lies between the locations of the perturbations at $\theta=22^{\circ}$.

The expression for determining $\kappa \omega_{2}$ is determined by considering the $\epsilon^{2}$ terms (Eq. (17)) and using the fact that $\mathbf{v}_{0_{2}}=\mathbf{v}_{0_{4}}=\mathbf{0}$,

$$
\left[\begin{array}{llll}
\mathbf{A}_{0_{1}} & \mathbf{A}_{0_{2}} & -\mathbf{A}_{0_{1}} & \mathbf{A}_{0_{4}}
\end{array}\right]\left[\begin{array}{c}
\mathbf{v}_{2_{1}} \\
\mathbf{v}_{2_{2}} \\
\mathbf{v}_{2_{3}} \\
\mathbf{v}_{2_{4}}
\end{array}\right]+\left[\begin{array}{llll}
\mathbf{A}_{1_{1}} & \mathbf{A}_{1_{2}} & \mathbf{A}_{1_{3}} & \mathbf{A}_{1_{4}}
\end{array}\right]\left[\begin{array}{c}
\tilde{\mathbf{v}}_{1_{1}} \\
\tilde{\mathbf{v}}_{1_{2}} \\
\tilde{\mathbf{v}}_{1_{3}} \\
\tilde{\mathbf{v}}_{1_{4}}
\end{array}\right]+\left(\mathbf{A}_{2_{1}}+\mathbf{A}_{2_{3}}\right) \tilde{\mathbf{v}}_{0_{1}}=\mathbf{0}
$$

which is rearranged to

$$
\begin{gathered}
{\left[\begin{array}{llll}
\left(\mathbf{A}_{1_{1}}+\mathbf{A}_{1_{3}}\right) & \mathbf{A}_{0_{2}} & \mathbf{A}_{0_{1}} & \mathbf{A}_{0_{4}}
\end{array}\right]\left[\begin{array}{c}
\tilde{\mathbf{v}}_{1_{3}} \\
\mathbf{v}_{2_{2}} \\
\mathbf{v}_{2_{1}}-\mathbf{v}_{2_{3}} \\
\mathbf{v}_{2_{4}}
\end{array}\right]} \\
+\left[\begin{array}{llll}
\left(\mathbf{A}_{2_{1}}+\mathbf{A}_{2_{3}}\right) & \mathbf{A}_{1_{2}} & \mathbf{A}_{1_{1}} & \mathbf{A}_{1_{4}}
\end{array}\right]\left[\begin{array}{c}
\tilde{\mathbf{v}}_{1_{1}} \\
\tilde{\mathbf{v}}_{1_{2}} \\
\tilde{\mathbf{v}}_{1_{1}}-\tilde{\mathbf{v}}_{1_{3}} \\
\tilde{\mathbf{v}}_{1_{4}}
\end{array}\right]=\mathbf{0}
\end{gathered}
$$

The left-most matrix is rank 11 at $\left\{\kappa \omega_{1_{1}}, \kappa \omega_{1_{2}}\right\}$ and the orthogonal complement of its range is spanned by the vector $\mathbf{P}_{1} \in \mathbf{R}^{12}$. Left-multiplying Eq. (38) by $\mathbf{P}_{1}^{T}$ yields a scalar expression involving $\kappa \omega_{2}$,

$$
\mathbf{P}_{1}^{T}\left[\left(\mathbf{A}_{2_{1}}+\mathbf{A}_{2_{3}}\right) \quad \mathbf{A}_{1_{2}} \quad \mathbf{A}_{1_{1}} \quad \mathbf{A}_{1_{4}}\right]\left[\begin{array}{c}
\tilde{\mathbf{v}}_{1_{1}} \\
\tilde{\mathbf{v}}_{1_{2}} \\
\tilde{\mathbf{v}}_{1_{1}}-\tilde{\mathbf{v}}_{1_{3}} \\
\tilde{\mathbf{v}}_{1_{4}}
\end{array}\right]=\mathbf{0}
$$

Evaluating Eq. (39) at $\kappa \omega_{1_{1}}$ yields $\omega_{2_{1}}=9.2098$, and at $\kappa \omega_{1_{2}}$ yields $\kappa \omega_{2_{2}}=6.5597$. The process for solving the equation associated with $\epsilon^{3}$ is similar to the single mass perturbation case and leads 
to analysis of

$$
\begin{aligned}
& {\left[\begin{array}{llll}
\left(\mathbf{A}_{3_{1}}+\mathbf{A}_{33}\right) & \mathbf{A}_{22} & \mathbf{A}_{21} & \mathbf{A}_{2_{4}}
\end{array}\right]\left[\begin{array}{c}
\tilde{\mathbf{v}}_{1_{1}} \\
\tilde{\mathbf{v}}_{1_{2}} \\
\tilde{\mathbf{v}}_{1_{1}}-\tilde{\mathbf{v}}_{1_{3}} \\
\tilde{\mathbf{v}}_{1_{4}}
\end{array}\right]+} \\
& {\left[\begin{array}{llll}
\left(\mathbf{A}_{2_{1}}+\mathbf{A}_{2_{3}}\right) & \mathbf{A}_{1_{2}} & \mathbf{A}_{1_{1}} & \mathbf{A}_{1_{4}}
\end{array}\right]\left[\begin{array}{c}
\tilde{\mathbf{v}}_{1_{3}} \\
\tilde{\mathbf{v}}_{2_{2}} \\
\tilde{\mathbf{v}}_{2_{1}}-\tilde{\mathbf{v}}_{2_{3}} \\
\tilde{\mathbf{v}}_{2_{4}}
\end{array}\right]+} \\
& {\left[\begin{array}{llll}
\left(\mathbf{A}_{1_{1}}+\mathbf{A}_{1_{3}}\right) & \mathbf{A}_{0_{2}} & \mathbf{A}_{0_{1}} & \mathbf{A}_{0_{4}}
\end{array}\right]\left[\begin{array}{c}
\tilde{\mathbf{v}}_{2_{3}} \\
\tilde{\mathbf{v}}_{3_{2}} \\
\tilde{\mathbf{v}}_{3_{1}}-\tilde{\mathbf{v}}_{3_{3}} \\
\tilde{\mathbf{v}}_{3_{4}}
\end{array}\right]=\mathbf{0}}
\end{aligned}
$$

Left-multiplication of Eq. (40) by $\mathbf{P}_{1}^{T}$ yields a scalar expression for $\kappa \omega_{3}$ because $\mathbf{P}_{1}$ is orthogonal to the ranges of the latter two matrices multiplying the coefficient vectors in Eq. (40). Completing this analysis yields the following frequency expansions

$$
\begin{aligned}
& \kappa \omega=\sqrt{\frac{36}{5}}+\epsilon(-2.7395)+\epsilon^{2}(9.2098)+\epsilon^{3}(-30.726)+\cdots, \\
& \kappa \omega=\sqrt{\frac{36}{5}}+\epsilon(-2.6271)+\epsilon^{2}(6.5597)+\epsilon^{3}(-24.643) \cdots .
\end{aligned}
$$

Table 1 summarizes the expansions for additional values of $\theta_{0}$. It is also evident from the table that as $\theta_{0}$ approaches $45^{\circ}$, the difference between $\kappa \omega_{1_{1}}$ and $\kappa \omega_{1_{2}}$ approaches zero, thus, identical mass perturbations at $\theta=\left\{0^{\circ}, 45^{\circ}\right\}$ only weakly detune the modal frequencies.

\subsubsection{Identical masses at $\theta=0^{\circ}$ and $\theta_{0}=45^{\circ}$}

The $\theta_{0}=45^{\circ}$ case is considered separately because there is an additional reduction in rank in the $\epsilon^{1}$-level analysis and so additional steps are required in order to resolve the weight vectors. 
Since $\operatorname{rank}\left(\mathbf{A}_{0}\right)=10, \mathbf{P}_{0}$ is still defined so that its columns span $\mathcal{R}\left(\mathbf{A}_{0}\right)^{\perp}$, however, Eq. (34) is now

$$
\left[\begin{array}{cc}
3.6795+1.3713 \kappa \omega_{1} & 35.047+13.061 \kappa \omega_{1} \\
35.047+13.061 \kappa \omega_{1} & -3.6795-1.3713 \kappa \omega_{1}
\end{array}\right] \mathbf{v}_{0_{1}}=\mathbf{0},
$$

which is singular at only one value of $\kappa \omega_{1}$, namely $\kappa \omega_{1_{1}}=\kappa \omega_{1_{2}}=-2.6833$. In this case the matrix multiplying $\mathbf{v}_{0_{1}}$ evaluates to zero. Thus, $\operatorname{dim} \mathcal{N}\left(\left[\left(\mathbf{A}_{1_{1}}+\mathbf{A}_{1_{3}}\right) \quad \mathbf{A}_{0_{2}} \quad \mathbf{A}_{0_{1}} \mathbf{A}_{0_{4}}\right]\right)=2$ in Eq. (35). Let $\mathbf{u}_{1}, \mathbf{u}_{2} \in \mathbf{R}^{12}$ form a basis for this null space and, furthermore, let the columns of $\mathbf{P}_{1} \in \mathbf{R}^{12 \times 2} \operatorname{span} \mathcal{R}\left(\left[\left(\begin{array}{llll}\left.\mathbf{A}_{1_{1}}+\mathbf{A}_{1_{3}}\right) & \mathbf{A}_{0_{2}} & \mathbf{A}_{0_{1}} & \mathbf{A}_{0_{4}}\end{array}\right]\right)^{\perp}\right.$. Solution vectors are then represented by

$$
\begin{array}{r}
\tilde{\mathbf{v}}_{0_{1}}=\alpha \mathbf{u}_{1_{1}}+\beta \mathbf{u}_{2_{1}} \\
\tilde{\mathbf{v}}_{1_{2}}=\alpha \mathbf{u}_{1_{2}}+\beta \mathbf{u}_{2_{2}} \\
\tilde{\mathbf{v}}_{1_{1}-\tilde{\mathbf{v}}_{1_{3}}}=\alpha \mathbf{u}_{1_{3}}+\beta \mathbf{u}_{2_{3}} \\
\tilde{\mathbf{v}}_{1_{4}}=\alpha \mathbf{u}_{1_{4}}+\beta \mathbf{u}_{2_{4}},
\end{array}
$$

where $\mathbf{u}_{1}$ and $\mathbf{u}_{2}$ are partitioned in the same manner as the solution vectors, and where $\alpha$ and $\beta$ represent real parameters which will be determined at the next stage of analysis. 
Left-multiplying Eq. (38) by $\mathbf{P}_{1}^{T}$ yields the analog of Eq. (39),

$$
\mathbf{P}_{1}^{T}\left[\left(\begin{array}{llll}
\left.\mathbf{A}_{2_{1}}+\mathbf{A}_{2_{3}}\right) & \mathbf{A}_{1_{2}} & \mathbf{A}_{1_{1}} & \mathbf{A}_{1_{4}}
\end{array}\right]\left[\begin{array}{l}
\alpha \mathbf{u}_{1_{1}}+\beta \mathbf{u}_{2_{1}} \\
\alpha \mathbf{u}_{1_{2}}+\beta \mathbf{u}_{2_{2}} \\
\alpha \mathbf{u}_{1_{3}}+\beta \mathbf{u}_{2_{3}} \\
\alpha \mathbf{u}_{1_{4}}+\beta \mathbf{u}_{2_{4}}
\end{array}\right]=\mathbf{0} .\right.
$$

This expression is rearranged to

$$
\begin{aligned}
\alpha \mathbf{P}_{1}^{T}\left(\mathbf{A}_{1_{2}} \mathbf{u}_{1_{2}}+\right. & \left.\mathbf{A}_{1_{3}} \mathbf{v}_{1_{1}}-\mathbf{A}_{1_{3}} \mathbf{u}_{1_{3}}+\left(\mathbf{A}_{2_{1}}+\mathbf{A}_{2_{3}}\right) \mathbf{u}_{1_{1}}\right) \\
& +\beta \mathbf{P}_{1}^{T}\left(\mathbf{A}_{1_{2}} \mathbf{u}_{2_{2}}+\mathbf{A}_{1_{3}} \mathbf{v}_{1_{1}}-\mathbf{A}_{1_{3}} \mathbf{u}_{2_{3}}+\left(\mathbf{A}_{2_{1}}+\mathbf{A}_{2_{3}}\right) \mathbf{u}_{2_{1}}\right)=\mathbf{0}
\end{aligned}
$$

When computing $\mathbf{u}_{1}, \mathbf{u}_{2}$ and $\mathbf{P}_{1}$ from a singular value decomposition of $\left[\left(\mathbf{A}_{1_{1}}+\mathbf{A}_{1_{3}}\right) \mathbf{A}_{0_{2}} \mathbf{A}_{0_{1}} \mathbf{A}_{0_{4}}\right]$, Eq. (42) can be rewritten as

$$
\left[\begin{array}{cc}
0.31427-0.080763 \kappa \omega_{2} & 4.7283-0.69671 \kappa \omega_{2} \\
3.0604-0.35681 \kappa \omega_{2} & 0.52256-0.11416 \kappa \omega_{2}
\end{array}\right]\left[\begin{array}{l}
\alpha \\
\beta
\end{array}\right]=\mathbf{0},
$$

which yields $\kappa \omega_{2_{1}}=6.6730$ and $\kappa \omega_{2_{2}}=8.9562$.

The eigenfunction weights are of interest for this perturbation scenario since the modal frequencies are degenerate to $\epsilon^{1}$. For $\kappa \omega_{2_{1}}, \alpha=0.33215$ and $\beta=0.94323$ from which the leading order terms in the weights are determined

$$
\tilde{\mathbf{v}}_{0}=\left[\begin{array}{ll}
-0.70711 & -0.70711
\end{array}\right]^{T}
$$

Similarly, for $\kappa \omega_{2_{2}}$, then $\alpha=-0.96528$ and $\beta=0.26122$ so

$$
\tilde{\mathbf{v}}_{0}=\left[\begin{array}{ll}
-0.70711 & 0.70711
\end{array}\right]^{T}
$$

Thus, the tangential displacements associated with the modes are dominated by $W(\theta)=\cos (2 \theta)+$ $\sin (2 \theta)$ and $W(\theta)=-\cos (2 \theta)+\sin (2 \theta)$, which implies the radial displacements with the slightly detuned modes will be proportional to $U(\theta) \propto-\sin (2 \theta)+\cos (2 \theta)$ and $U(\theta) \propto \sin (2 \theta)+\cos (2 \theta)$. The antinodes of the radial displacement subtend $45^{\circ}$ but one antinode at $\theta=22.5^{\circ}$ is located between the perturbation locations. 


\subsection{Mass-spring perturbation}

This section considers a mass perturbation located at $\theta=0^{\circ}$ and a radial spring perturbation located at $\theta=45^{\circ}$. The objective is to choose the spring parameter $g_{2}$ such that the first-order expansion values for the perturbed $n=2$ modal frequencies are equal, i.e. $\omega_{1_{1}}=\omega_{1_{2}}$ (the mass parameter $g_{1}$ is taken to be unity). This will guarantee that the $n=2$ modes will be essentially tuned for sufficiently small $\epsilon$, however, the behavior of the eigenfunction weight vector is of particular interest in this scenario. It will be shown that like the dual mass perturbation case, the leading order terms in the eigenfunction weight vector demonstrate that the anti-node orientations of the modes do not coincide with the $\left\{0^{\circ}, 45^{\circ}\right\}$ locations of the perturbations.

The nominal spring rate is the ring bending stiffness $K=E I / R^{3}$. A single radial spring affects only one modal frequency in the unperturbed ring. When $g_{1}=0$ and $g_{2}=1$, the following expansion parameters are derived,

$$
\kappa \omega_{1_{1}}=0 \quad, \quad \kappa \omega_{1_{2}}=0.047451
$$

where $\kappa \omega_{12}$ is associated with the perturbed mode whose radial displacement antinode is aligned with the spring at $\theta=45^{\circ}$. On the other hand, a single mass perturbation at $\theta=0^{\circ}$ decreases both modal frequencies (see Eq. (21)),

$$
\kappa \omega_{1_{1}}=-2.1466 \quad, \quad \kappa \omega_{1_{2}}=-0.53665 .
$$

The perturbations of the modal frequencies are additive at this order, so a simultaneous perturbation with a mass located at $\theta=0^{\circ}$ and a radial spring at $\theta=45^{\circ}$ yields

$$
\begin{aligned}
& \kappa \omega_{1_{1}}=-2.1466+0 g_{2} \\
& \kappa \omega_{1_{2}}=-0.53665+0.047451 g_{2},
\end{aligned}
$$

where $g_{1}=1$ and $g_{2}$ remains a parameter. By selecting $g_{2}=-33.929$ (a negative spring stiffness), $\kappa \omega_{1_{1}}=\kappa \omega_{1_{2}}=-2.1466$, so for sufficiently small $\epsilon$ the modal frequencies can be considered degenerate. 
The weight vectors for the perturbed modes are of interest, however, analysis of $\mathbf{A}_{0} \mathbf{v}_{1}+\mathbf{A}_{1} \mathbf{v}_{0}=$ 0 yields $\kappa \omega_{1_{1}}=\kappa \omega_{1_{2}}=-2.1466$ as constructed above but not the leading-order terms in the weight vector, $\mathbf{v}_{0}$. In order to compute the weight vector, Eq. (35) is analyzed for this case. Since

$$
\operatorname{rank}\left(\left[\left(\mathbf{A}_{1_{1}}+\mathbf{A}_{1_{3}}\right) \quad \mathbf{A}_{0_{2}} \mathbf{A}_{0_{1}} \mathbf{A}_{0_{4}}\right]\right)=10
$$

then $\mathbf{u}_{1}, \mathbf{u}_{2} \in \mathbf{R}^{12}$ are defined as a basis for $\mathcal{N}\left(\left[\left(\mathbf{A}_{1_{1}}+\mathbf{A}_{1_{3}}\right) \quad \mathbf{A}_{0_{2}} \quad \mathbf{A}_{0_{1}} \mathbf{A}_{0_{4}}\right]\right)$. The solution vector is parameterized using the same form as Eq. (41) but only the first partitions are reported because they determine $\tilde{\mathbf{v}}_{0_{1}}$,

$$
\tilde{\mathbf{v}}_{0_{1}}=\alpha \mathbf{u}_{1_{1}}+\beta \mathbf{u}_{2_{1}}, \quad \mathbf{u}_{1_{1}}=\left[\begin{array}{c}
-0.99856 \\
0.053726
\end{array}\right], \quad \mathbf{u}_{1_{1}}=\left[\begin{array}{l}
0.36301 \\
0.93179
\end{array}\right] .
$$

Furthermore, Eq. (38) reduces to

$$
\left[\begin{array}{cc}
-7.8786+8.0818 \kappa \omega_{2} & 26.956-12.359 \kappa \omega_{2} \\
44.258-10.352 \kappa \omega_{2} & 30.634-4.4431 \kappa \omega_{2}
\end{array}\right]\left[\begin{array}{l}
\alpha \\
\beta
\end{array}\right]=\mathbf{0}
$$

so the following values for $\kappa \omega_{2}$ and $\{\alpha, \beta\}$ are determined,

$$
\kappa \omega_{2_{1}}=1.7427, \quad\{\alpha, \beta\}=\{-0.65770,0.75328\} \Longrightarrow \tilde{\mathbf{v}}_{0_{1}}=\left[\begin{array}{l}
0.81285 \\
0.58247
\end{array}\right]
$$

and

$$
\kappa \omega_{2_{1}}=5.0235, \quad\{\alpha, \beta\}=\{0.73174,0.68158\} \Longrightarrow \tilde{\mathbf{v}}_{0_{1}}=\left[\begin{array}{c}
-0.58247 \\
0.81285
\end{array}\right],
$$

where the $\tilde{\mathbf{v}}_{0_{1}}$ are normalized to unit norm. This result demonstrates that for arbitrarily small but non-zero $\epsilon$, the leading order terms in the eigenfunction weights are orthogonal, which implies the antinodes associated with the modes subtend $45^{\circ}$, however, the antinodes are not aligned with the perturbation locations. The tangential displacements are dominated by the these leading order terms and are given by $W(\theta)=0.81285 \cos (2 \theta)+0.58247 \sin (2 \theta)$ and $W(\theta)=$ $-0.58247 \cos (2 \theta)+0.81285 \sin (2 \theta)$. Thus, the radial displacements $U$ will be proportional to

$$
\begin{aligned}
& U(\theta) \propto-0.81285 \sin (2 \theta)+0.58247 \cos (2 \theta)=\cos (2(\theta+27.2 \pi / 180)) \\
& U(\theta) \propto 0.58247 \sin (2 \theta)+0.81285 \cos (2 \theta)=\cos (2(\theta-17.8 \pi / 180)) .
\end{aligned}
$$




\section{Comparison with other analyses}

The perturbation results are compared to Rayleigh-Ritz analysis when the basis functions are selected to be $W(\theta)=\sum_{k}\left(\alpha_{k} \cos (k \theta)+\beta_{k} \sin (k \theta)\right)$, for $k=2$ (two-term), and for $k=$ $1, \ldots, 10$ (twenty-term). These basis functions satisfy the essential boundary conditions (continuity up to second derivative of the tangential displacement). The objective is to minimize the Lagrangian functional $\mathcal{L}$ with respect to basis function coefficients [21]. The resulting generalized eigenvalue problem is numerically solved. Comparisons with finite element analysis of a thin silicon ring are also reported. The ring parameters are: $E=170 \mathrm{GPa}, \rho=2329 \mathrm{~kg} \mathrm{~m}^{-3}$, ring height $x=270 \mu \mathrm{m}$, ring width $y=100 \mu \mathrm{m}, I=1 / 12 x y^{3}, A=x y, R=0.5 \mathrm{~cm}$, and $\kappa=1.0137 \times 10^{-4} \mathrm{~s}$. Three-dimensional free tetrahedral elements are used and generate over one hundred thousand degrees of freedom. The density of a small plug of material is manipulated to create a localized change in mass. The added mass is normalized with respect to the nominal ring mass to define $\epsilon$.

The natural frequencies for the single added mass as a function of $\epsilon$ are depicted in Fig. 2 when $n=2,3$. The perturbation analysis, FEA and twenty-term Rayleigh-Ritz analysis produce essentially the same results for both $n=2$ and $n=3$ cases. The dual mass perturbation results are summarized in Figs. 3, 4 and 5 for the $n=2$ pair of modes. In these figures, the absolute value of the difference between the perturbed modal frequencies as a function of $\epsilon$ are shown when identical mass perturbations subtend $43^{\circ}, 44^{\circ}$ and $45^{\circ}$. The modes weakly detune in these cases, however, for sufficiently large mass the perturbation expressions show closer agreement with the FEA. The results also depict interesting behavior for the dual mass perturbations when the subtended angle is not equal to $45^{\circ}$ : the existence of a non-zero perturbation at which the modal frequencies are equal. The finite perturbation that achieves degeneracy is quite accurately predicted by the perturbation analysis as seen in Figs. 3 and 4. Lastly, Fig. 6 shows the normalized modal frequency difference as a function of $\epsilon$ for the spring-mass perturbation. It can be shown that the two-term Rayleigh-Ritz analysis produces the same order- $\epsilon$ dependence as the perturbation analysis for these case studies. Thus, for sufficiently small perturbations, the two-term Rayleigh-Ritz analysis offers 
Figure 2: Top: The modal frequencies when $n=2$ modes as a function of a single mass perturbation. Bottom: The modal frequencies when $n=3$ modes as a function of a single mass perturbation.

greater simplicity for predicting the perturbed modal frequencies. This simplicity is advantageous when deriving a trimming procedure for reducing the modal frequency difference in ring resonators as described in [5]. Although the perturbation analysis appears to show greater predictive capability for large perturbations, especially with multiple masses, an effective trimming procedure based these results is still to be developed.

The discrepancy between the perturbation results and Rayleigh-Ritz analysis for large perturbations can be attributed to the fact that the third and fourth derivatives of the radial displacement 


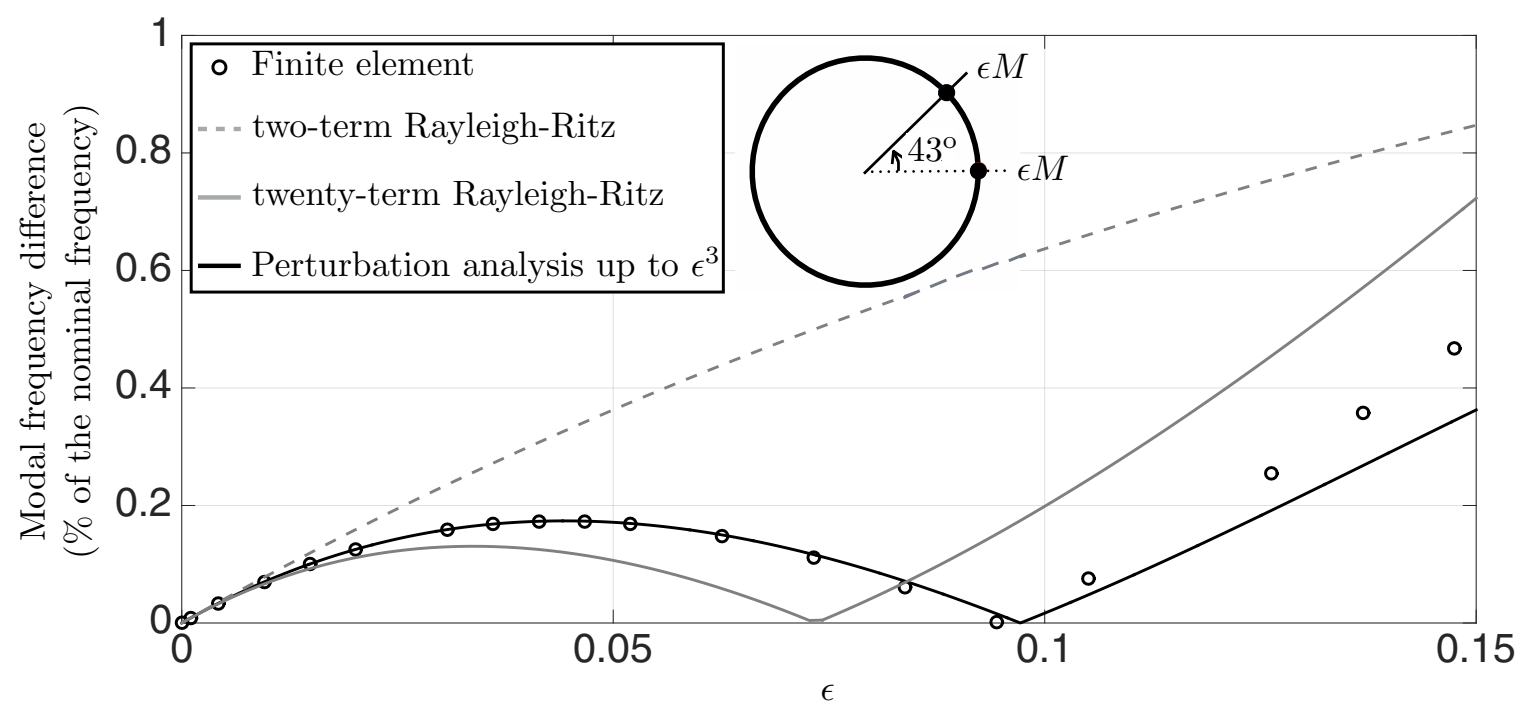

Figure 3: The normalized frequency split of the $n=2$ modal frequencies with respect to $\epsilon$ when $\theta_{0}=43^{\circ}$.

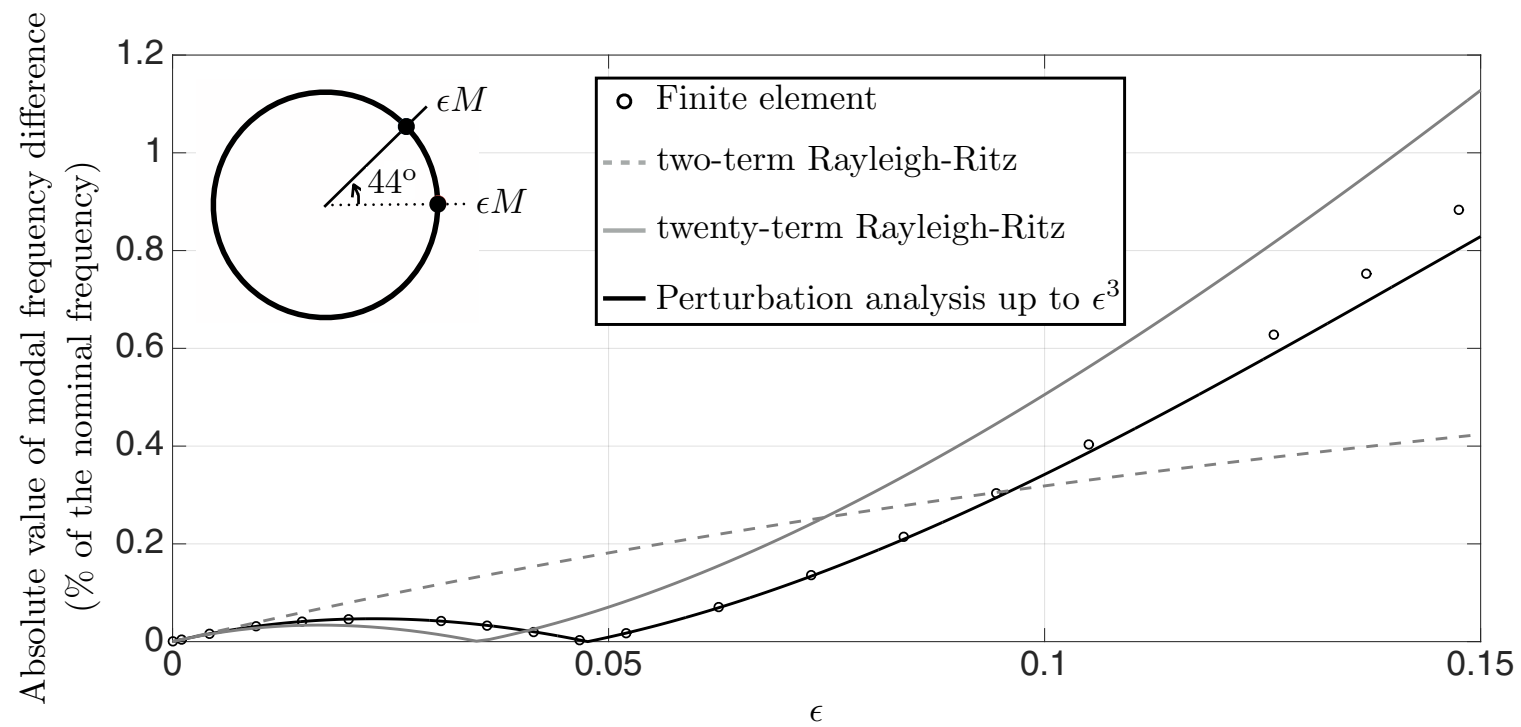

Figure 4: The normalized frequency split of the $n=2$ modal frequencies with respect to $\epsilon$ when $\theta_{0}=44^{\circ}$. 


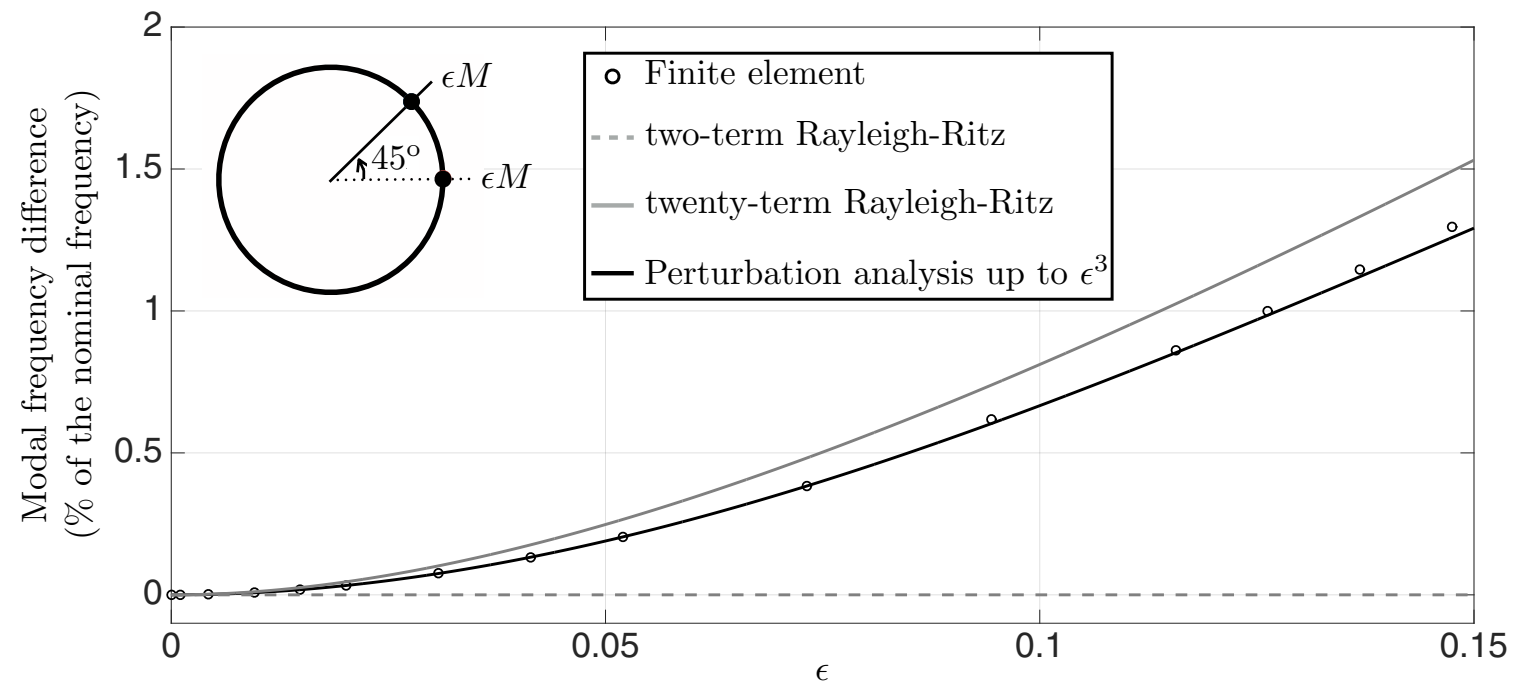

Figure 5: The normalized frequency split of the $n=2$ modal frequencies with respect to $\epsilon$ when $\theta_{0}=45^{\circ}$. The modal frequencies detune for a non-zero perturbation.

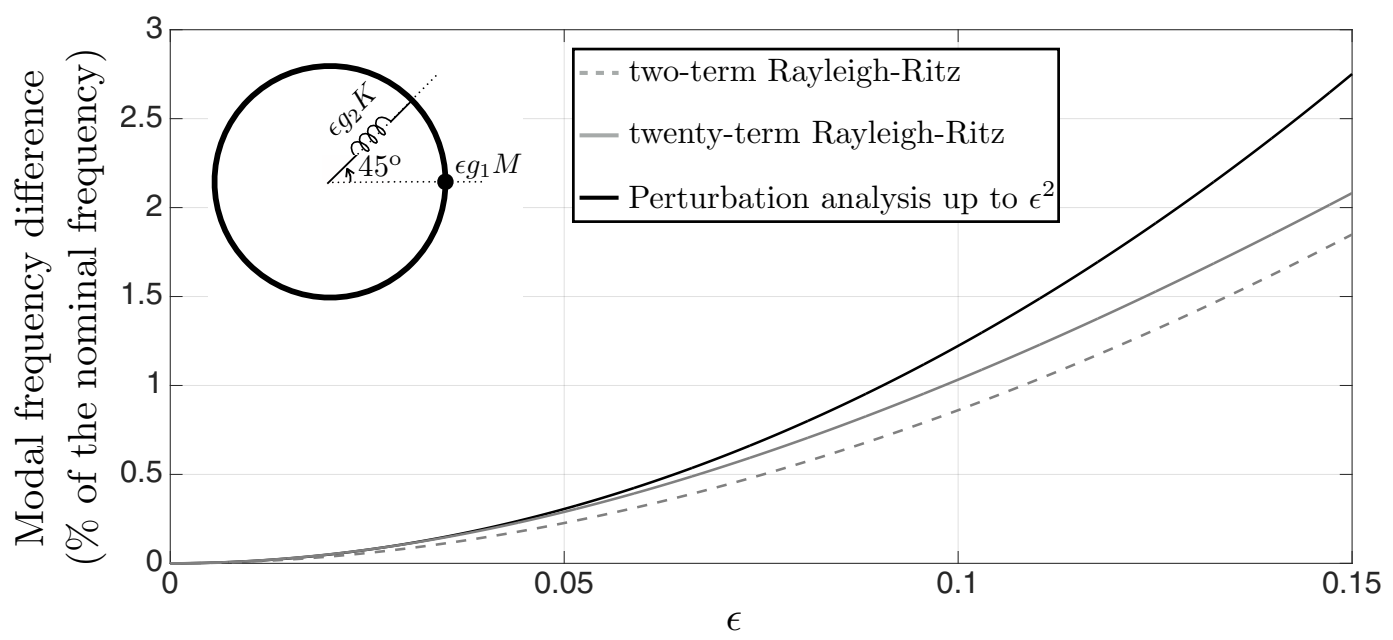

Figure 6: The normalized frequency split of the $n=2$ modal frequencies with respect to $\epsilon$ for a simultaneous massspring perturbation. 
with respect to $\theta$ are not continuous because of the boundary conditions in Eq. (5) (equivalent to the fourth and fifth derivatives with respect $\theta$ for the tangential displacement $W$ ). The discontinuity is proportional to $\epsilon$ and is essentially "built in" the perturbation solution. As the perturbation magnitude increases, the discontinuity becomes more severe, and the Rayleigh-Ritz basis functions, which have continuous derivatives of all orders have greater difficulty in approximating $W^{(4)}$ and $W^{(5)}$ in a neighborhood of the perturbation locations.

\section{Conclusion}

Perturbation expansions of the solutions of an imperfect ring have been developed for several case studies when the perturbations are constrained to be point masses and massless radial springs. Although each perturbation scenario is solved on a case-by-case basis, a general procedure is outlined for determining the expressions that yield the successive terms in the frequency expansions. Boundary conditions are determined that must be satisfied by the uniform ring segments that lie between the perturbation locations and the motion of these segments is represented as a weighted sum of the eigenfunctions for the uniform thin ring. The eigenfunctions, natural frequencies and weights are all functions of the perturbation parameter, $\epsilon$, which is the perturbing mass normalized by the ring mass. The expansions yield successive algebraic problems that are solved for a single perturbing mass, two masses with a varying angle between them, and a mass-spring perturbation. Rayleigh-Ritz analysis of the case studies using two- and twenty-term bases are also reported. For mass perturbations less than a few percent of the ring mass, all techniques yield essentially the same results, however, for larger mass ratios, the perturbation analysis more closely follows the finite element results in the two-mass cases.

\section{Acknowledgments}

The first author wishes to thank Prof. Stanley Dong. This research was partially supported by DARPA grant W31P4Q-11-1-0004. 


\section{Appendix A. Derivation of boundary conditions}

Hamilton's principle is applied to the Lagrangian $\mathcal{L}$ in order to derive the boundary conditions created by a point mass and radial spring perturbation co-located at $\theta=0^{\circ}$. The variation of $\mathcal{L}$ is summarized below,

$$
\begin{aligned}
& \int_{t_{1}}^{t_{2}}\left[-\int_{0}^{2 \pi} \rho A R \ddot{w}(\theta, t) \delta(w(\theta, t)) d \theta\right. \\
& -\left.\rho A R \frac{\partial \ddot{w}}{\partial \theta}(\theta, t) \delta(w(\theta, t))\right|_{0} ^{2 \pi}+\int_{0}^{2 \pi} \rho A R \frac{\partial^{2} \ddot{w}}{\partial \theta^{2}}(\theta, t) \delta(w(\theta, t)) d \theta \\
& -\epsilon g_{1} M \ddot{w}(0, t) \delta(w(0, t))-\epsilon g_{1} M \frac{\partial \ddot{w}}{\partial \theta}(0, t) \delta\left(\frac{\partial w}{\partial \theta}(0, t)\right) \\
& -\frac{E I}{R^{3}}\left(\left.\frac{\partial^{3} w}{\partial \theta^{3}} \delta\left(\frac{\partial^{2} w}{\partial \theta^{2}}\right)\right|_{0} ^{2 \pi}-\left.\frac{\partial^{4} w}{\partial \theta^{4}} \delta\left(\frac{\partial w}{\partial \theta}\right)\right|_{0} ^{2 \pi}+\left.\frac{\partial^{5} w}{\partial \theta^{5}} \delta(w)\right|_{0} ^{2 \pi}-\int_{0}^{2 \pi} \frac{\partial^{6} w}{\partial \theta^{6}} \delta(w) d \theta\right) \\
& -\frac{E I}{R^{3}}\left(\left.\frac{\partial^{3} w}{\partial \theta^{3}} \delta(w)\right|_{0} ^{2 \pi}-\int_{0}^{2 \pi} \frac{\partial^{4} w}{\partial \theta^{4}} \delta(w) d \theta\right) \\
& -\frac{E I}{R^{3}}\left(\left.\frac{\partial w}{\partial \theta} \delta\left(\frac{\partial^{2} w}{\partial \theta^{2}}\right)\right|_{0} ^{2 \pi}-\left.\frac{\partial^{2} w}{\partial \theta^{2}} \delta\left(\frac{\partial w}{\partial \theta}\right)\right|_{0} ^{2 \pi}+\left.\frac{\partial^{3} w}{\partial \theta^{3}} \delta(w)\right|_{0} ^{2 \pi}-\int_{0}^{2 \pi} \frac{\partial^{4} w}{\partial \theta^{4}} \delta(w) d \theta\right) \\
& -\frac{E I}{R^{3}}\left(\left.\frac{\partial w}{\partial \theta} \delta(w)\right|_{0} ^{2 \pi}-\int_{0}^{2 \pi} \frac{\partial^{2} w}{\partial \theta^{2}} \delta(w) d \theta\right) \\
& \left.-\epsilon g_{2} k \frac{\partial w}{\partial \theta}(0, t) \delta\left(\frac{\partial w}{\partial \theta}(0, t)\right)\right] d t=0
\end{aligned}
$$

By grouping terms associated with $\delta w(\theta, t)$, fundamental lemma of calculus of variables yields the equation of motion for the ring given in Eq. (3), which is equivalent to the equation of motion derived from the Newtonian approach [19].

The continuity of the radial and tangential motion and their derivatives at $\theta=0$ or $\theta=2 \pi$ dictate the continuity of $u(\theta, t), \frac{\partial u}{\partial \theta}(\theta, t), w(\theta, t)$ and $\frac{d w}{d \theta}(\theta, t)$ at that point so there are three essential boundary conditions

$$
\begin{aligned}
w(2 \pi, t) & =w(0, t), \\
\frac{\partial w}{\partial \theta}(2 \pi, t) & =\frac{\partial w}{\partial \theta}(0, t), \\
\frac{\partial^{2} w}{\partial \theta^{2}}(2 \pi, t) & =\frac{\partial^{2} w}{\partial \theta^{2}}(0, t) .
\end{aligned}
$$


The remaining natural boundary conditions are derived from Eq. (A.1) by gathering various terms. Noting $\delta w(0, t)=\delta w(2 \pi, t)$ yields

$$
-\epsilon g_{1} M \ddot{w}(0, t)-\frac{E I}{R^{3}}\left(\frac{\partial^{5} w}{\partial \theta^{5}}(2 \pi, t)-\frac{\partial^{5} w}{\partial \theta^{5}}(0, t)\right)-\frac{2 E I}{R^{3}}\left(\frac{\partial^{3} w}{\partial \theta^{3}}(2 \pi, 0)-\frac{\partial^{3} w}{\partial \theta^{3}}(0, t)\right)=0 .
$$

Furthermore, $\frac{\partial w}{\partial \theta}(0, t)=\frac{\partial w}{\partial \theta}(2 \pi, t)$ implies

$$
-\epsilon g_{1} M \frac{\partial \ddot{w}}{\partial \theta}(0, t)-\epsilon g_{2} k \frac{\partial w}{\partial \theta}(0, t)+\frac{E I}{R^{3}}\left(\frac{\partial^{4} w}{\partial \theta^{4}}(2 \pi, t)-\frac{\partial^{4} w}{\partial \theta^{4}}(0, t)\right)=0,
$$

and $\frac{\partial^{2} w}{\partial \theta^{2}}(0, t)=\frac{\partial^{2} w}{\partial \theta^{2}}(2 \pi, t)$ implies

$$
\frac{\partial^{3} w}{\partial \theta^{3}}(2 \pi, t)-\frac{\partial^{3} w}{\partial \theta^{3}}(0, t)=0
$$

Finally, substituting Eq. (A.7) into Eq. (A.5), simplifies the boundary condition involving the $5^{\text {th }}$ derivative of the tangential motion

$$
-\epsilon g_{1} M \ddot{w}(0, t)-\frac{E I}{R^{3}}\left(\frac{\partial^{5} w}{\partial \theta^{5}}(2 \pi, t)-\frac{\partial^{5} w}{\partial \theta^{5}}(0, t)\right)=0 .
$$

In summary, the six boundary conditions are given by Eqs. (A.2), (A.3), (A.4), (A.6), (A.7) and (A.8). 


\section{Appendix B. Expansions for $a, b$ and $c$ parameters when $n>2$}

\begin{tabular}{|c|c|c|}
\hline \multirow{3}{*}{$n=3$} & $a$ & $\begin{array}{l}3+\epsilon\left(0.16821 \kappa \omega_{1}\right)+\epsilon^{2}\left(0.16821 \kappa \omega_{2}-0.0048761 \kappa^{2} \omega_{1}^{2}\right) \\
+\epsilon^{3}\left(0.16821 \kappa \omega_{3}-0.0097522 \kappa^{2} \omega_{1} \omega_{2}+0.00029030 \kappa^{3} \omega_{1}^{3}\right)\end{array}$ \\
\hline & $b$ & $\begin{array}{l}2.4328+\epsilon\left(0.21262 \kappa \omega_{1}\right)+\epsilon^{2}\left(0.21262 \kappa \omega_{2}-0.010780 \kappa^{2} \omega_{1}^{2}\right) \\
+\epsilon^{3}\left(0.21262 \kappa \omega_{3}-0.021560 \kappa^{2} \omega_{1} \omega_{2}+0.0012651 \kappa^{3} \omega_{1}^{3}\right)\end{array}$ \\
\hline & $c$ & $\begin{array}{l}1.0399+\epsilon\left(-0.012171 \kappa \omega_{1}\right)+\epsilon^{2}\left(-0.012171 \kappa \omega_{2}+0.0029483 \kappa^{2} \omega_{1}^{2}\right) \\
+\epsilon^{3}\left(-0.012171 \kappa \omega_{3}+0.0058966 \kappa^{2} \omega_{1} \omega_{2}-0.00067236 \kappa^{3} \omega_{1}^{3}\right)\end{array}$ \\
\hline \multirow{3}{*}{$n=4$} & $a$ & $\begin{array}{l}4+\epsilon\left(0.049823 \kappa \omega_{1}\right)+\epsilon^{2}\left(0.049823 \kappa \omega_{2}+0.0027292 \kappa^{2} \omega_{1}^{2}\right) \\
+\epsilon^{3}\left(0.049823 \kappa \omega_{3}+0.0054584 \kappa^{2} \omega_{1} \omega_{2}-0.000070159 \kappa^{3} \omega_{1}^{3}\right)\end{array}$ \\
\hline & $b$ & $\begin{array}{l}3.6028+\epsilon\left(0.050394 \kappa \omega_{1}\right)+\epsilon^{2}\left(0.050394 \kappa \omega_{2}+0.0029990 \kappa^{2} \omega_{1}^{2}\right) \\
+\epsilon^{3}\left(0.050394 \kappa \omega_{3}+0.0059980 \kappa^{2} \omega_{1} \omega_{2}-0.000051990 \kappa^{3} \omega_{1}^{3}\right)\end{array}$ \\
\hline & $c$ & $\begin{array}{l}1.0098+\epsilon\left(-0.0029880 \kappa \omega_{1}\right)+\epsilon^{2}\left(-0.0029880 \kappa \omega_{2}+0.00071864 \kappa^{2} \omega_{1}^{2}\right) \\
+\epsilon^{3}\left(-0.0029880 \kappa \omega_{3}+0.0014373 \kappa^{2} \omega_{1} \omega_{2}-0.00016045 \kappa^{3} \omega_{1}^{3}\right)\end{array}$ \\
\hline \multirow{3}{*}{$n=5$} & $a$ & $\begin{array}{l}5+\epsilon\left(0.022966 \kappa \omega_{1}\right)+\epsilon^{2}\left(0.022966 \kappa \omega_{2}+0.0014391 \kappa^{2} \omega_{1}^{2}\right) \\
+\epsilon^{3}\left(0.022966 \kappa \omega_{3}+0.0028782 \kappa^{2} \omega_{1} \omega_{2}-0.0000098823 \kappa^{3} \omega_{1}^{3}\right)\end{array}$ \\
\hline & $b$ & $\begin{array}{l}4.6896+\epsilon\left(0.022918 \kappa \omega_{1}\right)+\epsilon^{2}\left(0.022918 \kappa \omega_{2}+0.0014626 \kappa^{2} \omega_{1}^{2}\right) \\
+\epsilon^{3}\left(0.022918 \kappa \omega_{3}+0.00292522 \kappa^{2} \omega_{1} \omega_{2}-0.0000067902 \kappa^{3} \omega_{1}^{3}\right)\end{array}$ \\
\hline & $c$ & $\begin{array}{c}1.0037+\epsilon\left(-0.0011189 \kappa \omega_{1}\right)+\epsilon^{2}\left(-0.0011189 \kappa \omega_{2}+0.00026885 \kappa^{2} \omega_{1}^{2}\right) \\
+\epsilon^{3}\left(-0.0011189 \kappa \omega_{3}+0.00053770 \kappa^{2} \omega_{1} \omega_{2}-0.000059818 \kappa^{3} \omega_{1}^{3}\right)\end{array}$ \\
\hline
\end{tabular}




\section{Appendix C. Expressions for the matrices in the single-mass analysis}

In this section, the numerical results associated with the intermediate steps for single mass perturbation analysis are presented. The partitions $\mathbf{A}_{0_{1}}$ and $\mathbf{A}_{0_{2}}$ are

$$
\mathbf{A}_{0_{1}}=\left[\begin{array}{ll}
0 & 0 \\
0 & 0 \\
0 & 0 \\
0 & 0 \\
0 & 0 \\
0 & 0
\end{array}\right], \quad \mathbf{A}_{0_{2}}=\left[\begin{array}{cccc}
384.45 & -232.32 & 383.45 & -232.32 \\
510.93 & -92.486 & 512.01 & -92.900 \\
592.24 & 110.65 & 591.24 & 111.54 \\
594.02 & 365.47 & 594.73 & 364.09 \\
492.47 & 639.47 & 492.28 & 641.26 \\
268.37 & 897.41 & 267.85 & 895.39
\end{array}\right] .
$$

Singular value decompositions are used to determine $\mathcal{R}(\cdot), \mathcal{R}(\cdot)^{\perp}$ and $\mathcal{N}(\cdot)$ where required. Analysis of $\mathbf{A}_{0}$ yields

$$
\mathbf{P}_{0}=\left[\begin{array}{cc}
-0.37374 & -0.50351 \\
0.50351 & -0.37374 \\
0.41527 & 0.55946 \\
-0.55946 & 0.41527 \\
-0.20763 & -0.27973 \\
0.27973 & -0.20763
\end{array}\right]
$$

For solving $\epsilon^{1}$ level equations, partitions of $\mathbf{A}_{1}$ are required,

$$
\mathbf{A}_{1_{1}}=\left[\begin{array}{cc}
0 & -1.6337 \kappa \omega_{1} \\
3.2674 \kappa \omega_{1} & 0 \\
0 & 6.5347 \kappa \omega_{1} \\
-13.069 \kappa \omega_{1} & 0 \\
0 & -26.139 \kappa \omega_{1}-90.478 \\
52.278 \kappa \omega_{1}+45.239 & 0
\end{array}\right]
$$


and

$$
\mathbf{A}_{1_{2}}=\left[\begin{array}{cccr}
299.02 \kappa \omega_{1} & -568.54 \kappa \omega_{1} & 299.02 \kappa \omega_{1} & -568.54 \kappa \omega_{1} \\
606.13 \kappa \omega_{1} & -582.20 \kappa \omega_{1} & 606.32 \kappa \omega_{1} & -582.08 \kappa \omega_{1} \\
985.84 \kappa \omega_{1} & -457.56 \kappa \omega_{1} & 985.33 \kappa \omega_{1} & -457.65 \kappa \omega_{1} \\
1383.8 \kappa \omega_{1} & -135.61 \kappa \omega_{1} & 1384.7 \kappa \omega_{1} & -135.78 \kappa \omega_{1} \\
1713.5 \kappa \omega_{1} & 426.28 \kappa \omega_{1}-18.697 & 1712.3 \kappa \omega_{1}-48.951 & 427.02 \kappa \omega_{1} \\
1848.0 \kappa \omega_{1}+45.239 & 1237.6 \kappa \omega_{1} & 1849.3 \kappa \omega_{1} & 1236.0 \kappa \omega_{1}
\end{array}\right] .
$$

The orthogonal complements of $\mathcal{R}\left(\left[\begin{array}{ll}\mathbf{A}_{1_{1}} & \mathbf{A}_{0_{2}}\end{array}\right]\right)$ are given by

$$
\mathbf{P}_{1}=\left[\begin{array}{llllll}
0 & 0.62706 & 0 & -0.69673 & 0 & 0.34837
\end{array}\right]^{T} \text { when } \kappa \omega_{1_{1}}=-2.1466 \quad \text { (C.5) }
$$

and

$$
\mathbf{P}_{1}=\left[\begin{array}{llllll}
0 & 0.62706 & 0 & -0.69673 & 0 & 0.34837
\end{array}\right]^{T} \text { when } \omega_{1_{2}}=-0.53666 .
$$

The first two columns of matrix $\mathbf{A}_{2}$ are grouped into one sub-matrix $\mathbf{A}_{2_{1}}$ and is function of the first two deviation terms in the frequency expansion $\kappa \omega_{1}$ and $\kappa \omega_{2}$.

$$
\mathbf{A}_{2_{1}}=\left[\begin{array}{cc}
2.6689\left(\kappa \omega_{1}\right)^{2} & 0.24103\left(\kappa \omega_{1}\right)^{2}-3.2674 \kappa \omega_{2} \\
0.36748\left(\kappa \omega_{1}\right)^{2}+6.5347 \kappa \omega_{2} & 5.3378\left(\kappa \omega_{1}\right)^{2} \\
-10.676\left(\kappa \omega_{1}\right)^{2} & 2.4340\left(\kappa \omega_{1}\right)^{2}+13.069 \kappa \omega_{2} \\
-8.2662\left(\kappa \omega_{1}\right)^{2}-26.139 \kappa \omega_{2} & -21.351\left(\kappa \omega_{1}\right)^{2} \\
42.703\left(\kappa \omega_{1}\right)^{2} & -23.329\left(\kappa \omega_{1}\right)^{2}-158.40 \kappa \omega_{1}-52.278 \kappa \omega_{2} \\
60.250\left(\kappa \omega_{1}\right)^{2}+67.438 \kappa \omega_{1}+104.56 \kappa \omega_{2} & 85.405\left(\kappa \omega_{1}\right)^{2}
\end{array}\right]
$$

The partitions $\mathbf{A}_{2_{2}}$ and $\mathbf{A}_{3_{1}}$ are necessary for computing the perturbed frequencies though $\epsilon^{3}$, but due to their lengthly nature, they are not included in this appendix.

\section{References}

[1] G. Bryan, On the beats in the vibrations of a revolving cylinder or bell, in: Proc. Cambridge Philosophical Society, Vol. VII, 1890, pp. 101-111. 
[2] A. E. H. Love, A treatise on the mathematical theory of elasticity, 1st Edition, Cambridge University press, 1892.

[3] T. Charnley, R. Perrin, Perturbation studies with a thin circular ring, Acustica 28 (3) (1973) $140-146$.

[4] D. Allaei, W. Soedel, T. Yang, Natural frequencies and modes of rings that deviate from perfect axisymmetry, J. Sound Vib. 111 (1) (1986) 9-27.

[5] C. Fox, A simple theory for the analysis and correction of frequency splitting in slightly imperfect rings, J. Sound Vib. 142 (2) (1990) 227-243.

[6] A. Rourke, S. McWilliam, C. Fox, Multi-mode trimming of imperfect rings, J. Sound Vib. 248 (4) (2001) 695-724.

[7] P. Bisegna, G. Caruso, Frequency split and vibration localization in imperfect rings, J. Sound Vib. 306 (3-5) (2007) 691-711.

[8] Y. Yoon, J. Lee, S. Yoo, H. Choi, A new method for in-plane vibration analysis of circular rings with widely distributed deviation, J. Sound Vib. 254 (4) (2002) 787-800.

[9] P. Shao, C. Mayberry, X. Gao, V. Tavassoli, F. Ayazi, A polysilicon microhemispherical resonating gyroscope, J. Microelectromech. Syst. 23 (2014) 762-764.

[10] I. Prikhodko, S. Zotov, A. Trusov, A. Shkel, Microscale glass-blown three-dimensional spherical shell resonators, J. Microelectromech. Sys. 20 (3) (2011) 691 - 701.

[11] S. Zotov, A. Trusov, A. Shkel, Three-dimensional spherical shell resonator gyroscope fabricated using wafer-scale glassblowing, J. Microelectromech. Syst. 21 (3) (2012) 509 - 510.

[12] J. Cho, J. Gregory, K. Najafi, High-Q, 3kHz single-crystal-silicon cylindrical rate-integrating gyro (CING), in: Proc. IEEE MEMS, Paris, 2012, pp. 172-175. 
[13] M. Kanik, P. Bordeenithikasem, D. Kim, N. Selden, A. Desai, R. M'Closkey, J. Schroers, Metallic glass hemispherical shell resonators, J. Microelectromech. Syst. 24 (1) (2015) 1928.

[14] J. Bernstein, M. Bancu, E. Cook, M. Chaparala, W. Teynor, M. Weinberg, A MEMS diamond hemispherical resonator, J. Micromech. Microeng. 23 (12) (2013) $1-8$.

[15] D. Schwartz, D. Kim, P. Stupar, J. DeNatale, R. M'Closkey, Modal parameter tuning of an axisymmetric resonator via mass perturbation, J. Microelectromech. Syst. 24 (3) (2015) 545555.

[16] D. Kim, A. Behbahani, P. Stupar, J. DeNatale, R. M'Closkey, Wafer-scale etch process for precision frequency tuning of MEMS gyros, in: Inertial Sensors and Systems (ISISS), 2015 IEEE International Symposium on, Hapuna Beach, Hawaii, 2015, pp. 1-2.

[17] P. Taheri-Tehrani, T.-H. Su, A. Heidari, G. Jaramillo, C. Yang, S. Akhbari, H. Najar, S. Nitzan, D. Saito, L. Lin, D. Horsley, Micro-scale diamond hemispherical resonator gyroscope, in: Proc. Solid-State Sensors, Actuators and Microsystems Workshop, Hilton Head, SC, 2014, pp. $289-292$.

[18] H. L. Langhaar, A strain-energy expression for thin elastic shells, Journal of Applied Mechanics (1949) 183-189.

[19] S. S. Rao, Vibration of Continuous Systems, John Wiley \& Sons, Hoboken, NJ, 2007.

[20] Wolfram Research, Inc., Mathematica, Version 10.4, Wolfram Research, Inc., Champaign, Illinois, 2016.

[21] L. Meirovitch, Analytical methods in vibrations, Macmillan series in advanced mathematics and theoretical physics, Macmillan, 1967. 